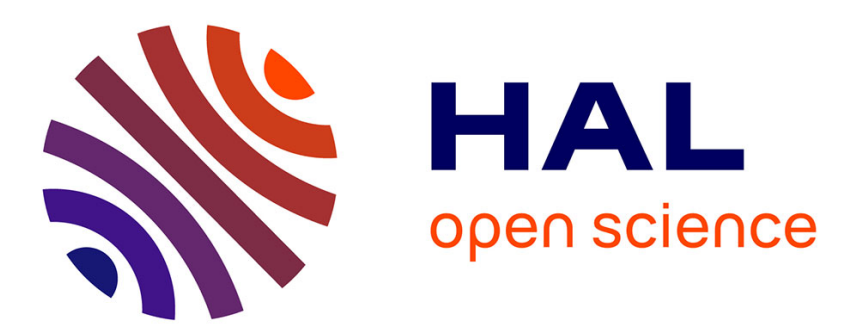

\title{
Homogenization of reactive flows in porous media and competition between bulk and surface diffusion
}

\author{
Grégoire Allaire, Harsha Hutridurga
}

\section{To cite this version:}

Grégoire Allaire, Harsha Hutridurga. Homogenization of reactive flows in porous media and competition between bulk and surface diffusion. IMA Journal of Applied Mathematics, 2012, 77, pp.788-815.

hal-00784041

\author{
HAL Id: hal-00784041 \\ https://hal.inria.fr/hal-00784041
}

Submitted on 5 Feb 2020

HAL is a multi-disciplinary open access archive for the deposit and dissemination of scientific research documents, whether they are published or not. The documents may come from teaching and research institutions in France or abroad, or from public or private research centers.
L'archive ouverte pluridisciplinaire HAL, est destinée au dépôt et à la diffusion de documents scientifiques de niveau recherche, publiés ou non, émanant des établissements d'enseignement et de recherche français ou étrangers, des laboratoires publics ou privés. 


\title{
Homogenization of reactive flows in porous media and competition between bulk and surface diffusion
}

\author{
G. Allaire *, H. Hutridurga *
}

\begin{abstract}
In this work, we study the convection and diffusion of a solute in a porous medium in the presence of a linear chemical reaction of adsorption/desorption on the pore surfaces. The mathematical model is a system of two coupled convection-diffusion equations, one in the bulk of the saturated fluid flowing in the porous medium, the other on the pore surface, at the interface with the solid part of the porous medium. The coupling takes place through a linear reaction term expressing the exchange of mass between the bulk concentration and the surface concentration. By a method of two-scale asymptotic expansion with drift we obtain the homogenized problem in a moving frame. We rigorously justify our upscaling approach by using the notion of two-scale convergence with drift. Some 2 -d numerical tests are performed in order to study the effect of variations of the adsorption rate constant and surface molecular diffusion on the effective dispersion tensor.
\end{abstract}

AMS classification: 35B27, 76M50

\section{Introduction}

Transport of solutes through porous media, where there is an exchange of mass at the interface between the bulk and the pore surface, has many applications in chemical engineering or in soil sciences [10], [16]. We understand porous media flows based on our knowledge of basic physical principles at the pore scale but most experimental observations are made at the macroscale. Direct numerical simulation for solving microscopic models are often impossible to handle with the available computational resources and thus upscaling is a necessary tool in practice. The method of multiscale asymptotic expansions is one of the most efficients to derive effective models at a macroscopic scale and has the definite advantage that it can be made mathematically rigorous through the theory of homogenization [17]. Dispersion phenomenon has attracted a lot of interest since the pioneering work of Taylor [29]. Multiple scale expansions were employed to study dispersion phenomena in presence of adsorption in many works including [4], [9], [12], [14], [18], [22], [23], [28] (and references therein).

In the present paper we consider a single solute in an incompressible saturated fluid flowing in a porous medium. A linear chemical reaction of adsorption/desorption can take place at the pore surfaces. Therefore, two unknown concentrations are taken into account: one in the bulk and the other on the liquid/solid interfaces. The mathematical model is a system of two coupled convection-diffusion equations, one in the bulk of the saturated fluid flowing in the porous medium, the other on the pores surface. This model is a generalization of that in [4], [6]: the novelty is the presence of convection and diffusion on the pores surface too (and

*Centre de Mathématiques Appliquées, CNRS UMR 7641, École Polytechnique, 91128 Palaiseau, France, gregoire.allaire@polytechnique.fr, hutridurga@cmap.polytechnique.fr 
not only in the bulk). In particular we study the possible competition between surface and bulk molecular diffusion. Higher values of the surface diffusion may favor a solute transport along the pore boundaries rather than in the bulk of the fluid. Of course, our model can still be considered as a toy model since in most applications one should consider multiple phase multi-component flow with more complex adsorption isotherms of Langmuir type. This will be the topic of future work [19].

Under a periodicity assumption on the porous media, our main result says that the homogenized or upscaled limit of both surface and bulk concentrations is given by the solution $\tilde{u}_{0}(t, x)$ of the following macroscopic convection-diffusion equation

$$
K_{d} \frac{\partial \tilde{u}_{0}}{\partial t}+\frac{1}{\varepsilon} b^{*} \cdot \nabla \tilde{u}_{0}-\operatorname{div}\left(A^{*} \nabla \tilde{u}_{0}\right)=0,
$$

where $K_{d}$ is the effective porosity, $A^{*}$ is the effective diffusion (or dispersion) tensor and $b^{*} / \varepsilon$ is the effective velocity. Note that, as usual, $\varepsilon$ is the small positive parameter which is the ratio between the heterogeneities lengthscale and a characteristic macroscopic lengthscale. Such a result is first obtained by the formal method of two-scale asymptotic expansion with drift (see [8], [4], although it was anticipated in [20], [26]) in Proposition 4.3 and Remark 4.4, which also deliver explicit formula for the effective coefficients. A mathematically rigorous proof of our main result is later given in Theorems 5.7 and 6.1 which rely on the notion of two-scale convergence with drift introduced in [21] (see [3] for a pedagogical review). This notion has also been applied to the homogenization of convection-diffusion equations in [8], [6], [13].

The contents of this paper is the following. Section 2 describes the periodic porous medium and the nondimensional microscopic model. Section 3 is devoted to uniform a priori estimates and briefly review existence results. In section 4 , we apply the formal method of two-scale asymptotic expansion with drift to deduce the homogenized limit. Section 5 is dedicated to the rigorous justification of the upscaling process and it contains most of the definitions and results of two-scale convergence theory. An improved convergence theorem (in the strong norm) is proved in Section 6. Eventually, Section 7 is concerned with some $2-d$ numerical tests using the FreeFem++ package [27]. In particular we study the behavior of the cell solutions and of the homogenized dispersion tensor with respect to variations of various microscopic parameters like the local Péclet number, the reaction rate and the surface molecular diffusion.

\section{Description of the problem}

We consider an $\varepsilon$-periodic infinite porous medium. Typically, this medium is built out of $\mathbb{R}^{d}$ ( $d=2$ or 3 , being the space dimension) by removing a periodic distribution of solid obstacles which, after rescaling, are all similar to the unit obstacle $\Sigma^{0}$. More precisely, let $Y=[0,1]^{d}$ be the unit periodicity cell. Let us consider a smooth partition $Y=\Sigma^{0} \cup Y^{0}$ where $\Sigma^{0}$ is the solid part and $Y^{0}$ is the fluid part. The unit periodicity cell is identified with the flat unit torus $\mathbb{T}^{d}$. The fluid part is assumed to be a smooth connected open subset whereas no particular assumptions are made on the solid part.

For each multi-index $j \in \mathbb{Z}^{d}$, we define $Y_{\varepsilon}^{j}=\varepsilon\left(Y^{0}+j\right), \Sigma_{\varepsilon}^{j}=\varepsilon\left(\Sigma^{0}+j\right), S_{\varepsilon}^{j}=$ $\varepsilon\left(\partial \Sigma^{0}+j\right)$, the periodic porous medium $\Omega_{\varepsilon}=\cup_{j \in \mathbb{Z}^{d}} Y_{\varepsilon}^{j}$ and the $(d-1)$-dimensional surface $\partial \Omega_{\varepsilon}=\cup_{j \in \mathbb{Z}^{d}} S_{\varepsilon}^{j}$.

We denote by $n(y)$ the exterior unit normal to $Y^{0}$. Then, $G(y)=I d-n(y) \otimes n(y)$ is the projection matrix on the tangent hyperplane to the surface $\partial Y^{0}=\partial \Sigma^{0}$. In order to define a Laplace-Beltrami operator on this surface, we define the tangential 
gradient $\nabla^{S}=G(y) \nabla$ and the tangential divergence $\operatorname{div}^{S} \Psi=\operatorname{div}(G(y) \Psi)$ for a vector field defined from $Y^{0}$ into $\mathbb{R}^{d}$.

We assume that the porous medium is saturated with an incompressible fluid, the velocity of which is assumed to be independent of time, periodic in space and given. The fluid cannot penetrate the solid obstacles but can slip on their surface. Therefore, we consider two periodic vector fields: $b(y)$, defined in the bulk $Y^{0}$, and $b^{S}(y)$, defined on the surface $\partial \Sigma^{0}$ and belonging at each point of $\partial \Sigma^{0}$ to its tangent hyperplane. Assuming that the fluid is incompressible and does not penetrate the obstacles means that

$$
\begin{gathered}
\operatorname{div}_{y} b(y)=0 \quad \text { in } Y^{0}, \quad b(y) \cdot n(y)=0 \quad \text { on } \partial \Sigma^{0}, \\
\operatorname{div}_{y}^{S} b^{S}(y)=0 \quad \text { on } \partial \Sigma^{0} .
\end{gathered}
$$

In truth, $b^{S}(y)$ should be the trace of $b(y)$ on $\partial \Sigma^{0}$ but, since this property is not necessary for our analysis, we shall not make such an assumption. Of course, some regularity is required for these vector fields and we assume that $b(y) \in L^{\infty}\left(Y^{0} ; \mathbb{R}^{d}\right)$, $b^{S}(y) \in L^{\infty}\left(\partial \Sigma^{0} ; \mathbb{R}^{d}\right)$ and $b^{S}=G b^{S}$ is always tangential to the surface.

We assume that the molecular diffusion is periodic, possibly anisotropic, varying in space and different in the bulk and on the surface. In other words, we introduce two periodic symmetric tensors $D(y)$ and $D^{S}(y)$, with entries belonging respectively to $L^{\infty}\left(Y^{0}\right)$ and to $L^{\infty}\left(\partial \Sigma^{0}\right)$, which are assumed to be uniformly coercive, namely there exists a constant $C>0$ such that, for any $\xi \in \mathbb{R}^{d}$,

$$
D(y) \xi \cdot \xi \geq C|\xi|^{2} \text { a.e. in } Y^{0}, \quad D^{S}(y) \xi \cdot \xi \geq C|\xi|^{2} \text { a.e. on } \partial \Sigma^{0} .
$$

Without loss of generality, we also assume that $D^{S}$ acts only on the tangent hyperplane of $\Sigma^{0}$, i.e., $D^{S}=G D^{S} G$.

Introducing the two positive constants $\kappa$ (the adsorption rate) and $K$ (the adsorption equilibrium constant), for some positive final time $T$ we consider the coupled system

$$
\begin{gathered}
\frac{\partial u_{\varepsilon}}{\partial t}+\frac{1}{\varepsilon} b_{\varepsilon} \cdot \nabla u_{\varepsilon}-\operatorname{div}\left(D_{\varepsilon} \nabla u_{\varepsilon}\right)=0 \text { in }(0, T) \times \Omega_{\varepsilon} \\
-\frac{D_{\varepsilon}}{\varepsilon} \nabla u_{\varepsilon} \cdot n=\frac{\partial v_{\varepsilon}}{\partial t}+\frac{1}{\varepsilon} b_{\varepsilon}^{S} \cdot \nabla^{S} v_{\varepsilon}-\operatorname{div}^{S}\left(D_{\varepsilon}^{S} \nabla^{S} v_{\varepsilon}\right)=\frac{\kappa}{\varepsilon^{2}}\left[u_{\varepsilon}-\frac{v_{\varepsilon}}{K}\right] \text { on }(0, T) \times \partial \Omega_{\varepsilon} \\
u_{\varepsilon}(0, x)=u^{0}(x) \text { in } \Omega_{\varepsilon}, v_{\varepsilon}(0, x)=v^{0}(x) \text { on } \partial \Omega_{\varepsilon}
\end{gathered}
$$

where the initial data are chosen such that $u^{0}(x) \in L^{2}\left(\mathbb{R}^{d}\right)$ and $v^{0}(x) \in H^{1}\left(\mathbb{R}^{d}\right)$ (so that its trace is well-defined on $\partial \Omega_{\varepsilon}$ ). The notations for the coefficients are, as usual, $D_{\varepsilon}(x)=D(x / \varepsilon), D_{\varepsilon}^{S}(x)=D^{S}(x / \varepsilon), b_{\varepsilon}(x)=b(x / \varepsilon)$ and $b_{\varepsilon}^{S}(x)=b^{S}(x / \varepsilon)$.

It should be noted here that the divergence free assumption and the no-penetration condition on the velocity field is not necessary as one can still homogenize the above system in their absence, using a factorization principle to start with as is done in [8].

The scaling of system (1)-(3), where some terms are very large of order $\varepsilon^{-1}$ or $\varepsilon^{-2}$, should not be a surprise for the attentive reader. Indeed, it is obtained from an adimensonalized system (without any $\varepsilon$ factor) by the parabolic change of variables $(\tau, y) \rightarrow\left(\varepsilon^{-2} t, \varepsilon^{-1} x\right)$. In other words, one can claim that, even though the global Péclet number is of the order of $\varepsilon^{-1}$, the local Péclet number is just of order 1 . We recall that the presence of a large drift is classical in the literature [4], [9], [13], [21], [22], [26], [28].

Remark 2.1 When $\kappa=0$, the equations for $u_{\varepsilon}$ and $v_{\varepsilon}$ are decoupled. So, we always assume $\kappa>0$. Formally, when $\kappa$ tends to $+\infty$, the two concentrations are fully coupled in the sense that $v_{\varepsilon}=K u_{\varepsilon}$. 
On the other hand, when $K=0$, it formally yields that $v_{\varepsilon}=0$ and the only remaining unknown $u_{\varepsilon}$ satisfies a homogeneous Neumann boundary condition on $\partial \Omega_{\varepsilon}$. However, if $K$ tends to $+\infty$, then $u_{\varepsilon}$ and $v_{\varepsilon}$ are again decoupled in the limit, with a Fourier-type boundary condition $-D_{\varepsilon} \nabla u_{\varepsilon} \cdot n=\kappa u_{\varepsilon} / \varepsilon$ on $\partial \Omega_{\varepsilon}$.

Remark 2.2 A crucial assumption in our work, as in [4] and[6], is that all coefficients are purely periodic functions depending only on the fast variable $y=x / \varepsilon$ and not on the slow variable $x$. In particular, we are unable to treat the case of more general velocity fields of the type $b_{\varepsilon}(x)=b(x, x / \varepsilon)$ and $b_{\varepsilon}^{S}(x)=b^{S}(x, x / \varepsilon)$ where $b(x, y)$ and $b^{S}(x, y)$ are smooth divergence-free (with respect to both variables) vector fields. The main technical reason is that the homogenized drift $b^{*}$ would then depend on $x$ which cannot be handled by our method. Even more, we know from [7] that, under special assumptions on the coefficients depending on $x$ and $y$, a new localization phenomenon can happen which is completely different from the asymptotic behavior proved in the present work. There is still a lot to understand for general velocity fields $b(x, y)$ and $b^{S}(x, y)$ but we are lacking the adequate tools (even formal ones) to guess the correct effective limit.

\section{A Priori Estimates}

The existence and uniqueness of solution to (1)-(3) is classical. One can either use the argument of Galerkin approximation followed by a priori estimates as in [15] (Chapter 7) or use a variant of the Lax-Milgram lemma due to Lions-Magenes (see, if necessary, chapter 10 in [11]).

The variational formulation for (1)-(2) amounts to find $\left(u_{\varepsilon}, v_{\varepsilon}\right)$ such that $u_{\varepsilon} \in$ $L^{2}\left((0, T) ; H^{1}\left(\Omega_{\varepsilon}\right)\right) \cap C\left([0, T] ; L^{2}\left(\Omega_{\varepsilon}\right)\right)$ and $v_{\varepsilon} \in L^{2}\left((0, T) ; H^{1}\left(\partial \Omega_{\varepsilon}\right)\right) \cap C\left([0, T] ; L^{2}\left(\partial \Omega_{\varepsilon}\right)\right)$ satisfying, for any test function $\left(\phi_{\varepsilon}, \psi_{\varepsilon}\right) \in H^{1}\left(\Omega_{\varepsilon}\right) \times H^{1}\left(\partial \Omega_{\varepsilon}\right)$,

$$
\begin{aligned}
& \int_{0}^{T} \int_{\Omega_{\varepsilon}}\left[\frac{\partial u_{\varepsilon}}{\partial t} \phi_{\varepsilon}+\frac{1}{\varepsilon} b_{\varepsilon} \cdot \nabla u_{\varepsilon} \phi_{\varepsilon}+D_{\varepsilon} \nabla u_{\varepsilon} \cdot \nabla \phi_{\varepsilon}\right] d x d t \\
& +\frac{\varepsilon}{K} \int_{0}^{T} \int_{\partial \Omega_{\varepsilon}}\left[\frac{\partial v_{\varepsilon}}{\partial t} \psi_{\varepsilon}+\frac{1}{\varepsilon} b_{\varepsilon}^{S} \cdot \nabla^{S} v_{\varepsilon} \psi_{\varepsilon}+D_{\varepsilon}^{S} \nabla^{S} v_{\varepsilon} \cdot \nabla^{S} \psi_{\varepsilon}\right] d \sigma_{\varepsilon}(x) d t \\
& +\int_{0}^{T} \int_{\partial \Omega_{\varepsilon}}\left[\frac{\kappa}{\varepsilon}\left(u_{\varepsilon}-\frac{v_{\varepsilon}}{K}\right)\left(\phi_{\varepsilon}-\frac{\psi_{\varepsilon}}{K}\right)\right] d \sigma_{\varepsilon}(x) d t=0 .
\end{aligned}
$$

From this variational formulation we deduce the following parabolic a priori estimate.

Lemma 3.1 There exists a constant $C$, which is independent of $\varepsilon$, such that the solution of (1)-(3) satisfies

$$
\begin{aligned}
& \left\|u_{\varepsilon}\right\|_{L^{\infty}\left((0, T) ; L^{2}\left(\Omega_{\varepsilon}\right)\right)}+\sqrt{\varepsilon}\left\|v_{\varepsilon}\right\|_{L^{\infty}\left((0, T) ; L^{2}\left(\partial \Omega_{\varepsilon}\right)\right)} \\
& +\left\|\nabla u_{\varepsilon}\right\|_{L^{2}\left((0, T) \times \Omega_{\varepsilon}\right)}+\sqrt{\varepsilon}\left\|\nabla^{S} v_{\varepsilon}\right\|_{L^{2}\left((0, T) \times \partial \Omega_{\varepsilon}\right)} \\
& +\sqrt{\varepsilon}\left\|w_{\varepsilon}\right\|_{L^{\infty}\left((0, T) ; L^{2}\left(\partial \Omega_{\varepsilon}\right)\right)} \leq C\left(\left\|u^{0}\right\|_{L^{2}\left(\mathbb{R}^{d}\right)}+\left\|v^{0}\right\|_{H^{1}\left(\mathbb{R}^{d}\right)}\right)
\end{aligned}
$$

where $w_{\varepsilon}=\varepsilon^{-1}\left(u_{\varepsilon}-K^{-1} v_{\varepsilon}\right)$. 
Remark 3.2 Since the $(d-1)$-dimensional measure of the periodic surface $\partial \Omega_{\varepsilon}$ is of the order of $\varepsilon^{-1}$, a bound of the type $\sqrt{\varepsilon}\left\|z_{\varepsilon}\right\|_{L^{2}\left(\partial \Omega_{\varepsilon}\right)} \leq C$ means that the sequence $z_{\varepsilon}$ is "bounded" on the surface $\partial \Omega_{\varepsilon}$.

Proof. We first derive an energy equality for (1)-(3). We multiply (1) by $u_{\varepsilon}$ and then integrate over $\Omega_{\varepsilon}$

$$
\frac{1}{2} \frac{d}{d t} \int_{\Omega_{\varepsilon}}\left|u_{\varepsilon}\right|^{2} d x+\int_{\Omega_{\varepsilon}} D_{\varepsilon} \nabla u_{\varepsilon} \cdot \nabla u_{\varepsilon} d x+\frac{\kappa \varepsilon}{\varepsilon^{2}} \int_{\partial \Omega_{\varepsilon}}\left(u_{\varepsilon}^{2}-\frac{u_{\varepsilon} v_{\varepsilon}}{K}\right) d \sigma_{\varepsilon}(x)=0
$$

where the integral of the convective term has disappeared since

$$
\int_{\Omega_{\varepsilon}} \frac{1}{\varepsilon} b_{\varepsilon} \cdot \nabla u_{\varepsilon} u_{\varepsilon} d x=\frac{1}{2} \int_{\Omega_{\varepsilon}} \operatorname{div}\left(b_{\varepsilon}\left|u_{\varepsilon}\right|^{2}\right) d x=\frac{1}{2} \int_{\partial \Omega_{\varepsilon}}\left|u_{\varepsilon}\right|^{2} b_{\varepsilon} \cdot n d \sigma_{\varepsilon}(x)=0 .
$$

Multiply the second equality in (2) by $K^{-1} \varepsilon v_{\varepsilon}$ and integrate over $\partial \Omega_{\varepsilon}$ to get

$$
\frac{\varepsilon}{2 K} \frac{d}{d t} \int_{\partial \Omega_{\varepsilon}}\left|v_{\varepsilon}\right|^{2} d \sigma_{\varepsilon}(x)+\frac{\varepsilon}{K} \int_{\partial \Omega_{\varepsilon}} D_{\varepsilon}^{S} \nabla^{S} v_{\varepsilon} \cdot \nabla^{S} v_{\varepsilon} d \sigma_{\varepsilon}(x)+\frac{\varepsilon \kappa}{\varepsilon^{2}} \int_{\partial \Omega_{\varepsilon}}\left(\frac{v_{\varepsilon}^{2}}{K^{2}}-\frac{u_{\varepsilon} v_{\varepsilon}}{K}\right) d \sigma_{\varepsilon}(x)=0
$$

where the integral of the convective term has also disappeared since $b_{\varepsilon}^{S}$ is divergence free. Adding (6) and (7) results in the following energy equality

$$
\begin{aligned}
& \frac{1}{2} \frac{d}{d t} \int_{\Omega_{\varepsilon}}\left|u_{\varepsilon}\right|^{2} d x+\frac{\varepsilon}{2 K} \frac{d}{d t} \int_{\partial \Omega_{\varepsilon}}\left|v_{\varepsilon}\right|^{2} d \sigma_{\varepsilon}(x)+\int_{\Omega_{\varepsilon}} D_{\varepsilon} \nabla u_{\varepsilon} \cdot \nabla u_{\varepsilon} d x \\
& +\frac{\varepsilon}{K} \int_{\partial \Omega_{\varepsilon}} D_{\varepsilon}^{S} \nabla^{S} v_{\varepsilon} \cdot \nabla^{S} v_{\varepsilon} d \sigma_{\varepsilon}(x)+\frac{\varepsilon \kappa}{\varepsilon^{2}} \int_{\partial \Omega_{\varepsilon}}\left(u_{\varepsilon}-\frac{v_{\varepsilon}}{K}\right)^{2} d \sigma_{\varepsilon}(x)=0
\end{aligned}
$$

Integrating it over the time interval $(0, T)$ leads to the desired estimate $(5)$.

\section{Two Scale Expansions with drift}

We homogenize the microscopic model (1)-(3) using the formal method of two-scale asymptotic expansions with drift [4], [6], [8], [13], [26]. This method starts with the assumption that $u_{\varepsilon}$ and $v_{\varepsilon}$ can be written in terms of an infinite series as

$$
u_{\varepsilon}(t, x)=\sum_{i=0}^{\infty} \varepsilon^{i} u_{i}\left(t, x-\frac{b^{*} t}{\varepsilon}, \frac{x}{\varepsilon}\right)
$$

and

$$
v_{\varepsilon}(t, x)=\sum_{i=0}^{\infty} \varepsilon^{i} v_{i}\left(t, x-\frac{b^{*} t}{\varepsilon}, \frac{x}{\varepsilon}\right)
$$

where $u_{i}(t, x, y)$ and $v_{i}(t, x, y)$ are functions of macroscopic variable $x$ and the microscopic variable $y=\frac{x}{\varepsilon} \in(0,1)^{d}$. The drift $b^{*} \in \mathbb{R}^{d}$ is unknown to start with. It shall be determined along the process of homogenization. The idea is to plug (8) and (9) in (1)-(2). We shall keep in mind the following chain rule differentiation:

$$
\begin{aligned}
& \frac{\partial}{\partial t}\left[\phi\left(t, x-\frac{b^{*} t}{\varepsilon}, \frac{x}{\varepsilon}\right)\right]=\left[\frac{\partial \phi}{\partial t}-\sum_{j=1}^{d} \frac{b_{j}^{*}}{\varepsilon} \frac{\partial \phi}{\partial x_{j}}\right]\left(t, x-\frac{b^{*} t}{\varepsilon}, \frac{x}{\varepsilon}\right), \\
& \frac{\partial}{\partial x_{j}}\left[\phi\left(t, x-\frac{b^{*} t}{\varepsilon}, \frac{x}{\varepsilon}\right)\right]=\left[\frac{\partial \phi}{\partial x_{j}}+\frac{1}{\varepsilon} \frac{\partial \phi}{\partial y_{j}}\right]\left(t, x-\frac{b^{*} t}{\varepsilon}, \frac{x}{\varepsilon}\right) .
\end{aligned}
$$


Inserting the asymptotic expansions of the solutions in the model, we identify the coefficients of the various powers of $\varepsilon$ to get a cascade of systems of equations. We present a lemma that plays a crucial role in solving those systems of pde's.

Lemma 4.1 For $f \in L^{2}\left(Y^{0}\right), g \in L^{2}\left(\partial \Sigma^{0}\right)$ and $h \in L^{2}\left(\partial \Sigma^{0}\right)$, the following system of equations

$$
\begin{cases}b(y) \cdot \nabla_{y} u-\operatorname{div}_{y}\left(D(y) \nabla_{y} u\right)=f & \text { in } Y^{0} \\ -D(y) \nabla_{y} u \cdot n+g=\kappa\left(u-\frac{1}{K} v\right) & \text { on } \partial \Sigma^{0} \\ b^{S}(y) \cdot \nabla_{y}^{S} v-\operatorname{div}_{y}^{S}\left(D^{S}(y) \nabla_{y}^{S} v\right)-h=\kappa\left(u-\frac{1}{K} v\right) & \text { on } \partial \Sigma^{0} \\ y \rightarrow(u(y), v(y)) & Y-\text { periodic }\end{cases}
$$

admits a solution $(u, v) \in H_{\#}^{1}\left(Y^{0}\right) \times H_{\#}^{1}\left(\partial \Sigma^{0}\right)$, unique up to the addition of a constant multiple of $(1, K)$, if and only if

$$
\int_{Y_{0}} f d y+\int_{\partial \Sigma_{0}}(g+h) d \sigma(y)=0 .
$$

Remark 4.2 The subscript \# indicates a space of $Y$-periodic functions. Recall that $Y^{0}$ and $\Sigma^{0}$ are thought as subsets of the unit cell $Y$, identified with the unit flat torus, i.e., $Y^{0}$ and $\Sigma^{0}$ are periodic subsets of $\mathbb{R}^{d}$.

Proof. The variational formulation of (11) is

$$
\begin{gathered}
\int_{Y^{0}}\left(b \cdot \nabla_{y} u\right) \phi d y+\int_{Y^{0}} D(y) \nabla_{y} u \cdot \nabla_{y} \phi d y+ \\
\frac{1}{K} \int_{\partial \Sigma^{0}}\left(b^{S} \cdot \nabla_{y}^{S} v\right) \psi d y+\frac{1}{K} \int_{\partial \Sigma^{0}} D^{S}(y) \nabla_{y}^{S} v \cdot \nabla_{y}^{S} \psi d \sigma(y)+ \\
\int_{\partial \Sigma^{0}} \kappa\left[u-\frac{v}{K}\right]\left[\phi-\frac{\psi}{K}\right] d \sigma(y)=\int_{Y_{0}} f \phi d y+\int_{\partial \Sigma_{0}}\left(g \phi+\frac{h}{K} \psi\right) d \sigma(y) .
\end{gathered}
$$

Taking $(\phi, \psi)=(1, K)$ we find the necessary condition (12). The left hand side of the variational formulation is coercive on the space $\left[H_{\#}^{1}\left(Y^{0}\right) \times H_{\#}^{1}\left(\partial \Sigma^{0}\right)\right] /[\mathbb{R}(1, K)]$ where the space $\mathbb{R}(1, K)$ is the set of constant vectors of the type $(C, K C)$ when $C$ takes values in $\mathbb{R}$. Lax-Milgram lemma yields the existence of a unique solution in this space.

Proposition 4.3 Under the assumption (8)-(9), the solution $\left(u_{\varepsilon}, v_{\varepsilon}\right)$ of (1)-(3) formally satisfy

$$
\begin{aligned}
& u_{\varepsilon}(t, x) \approx u_{0}\left(t, x-\frac{b^{*} t}{\varepsilon}\right)+\varepsilon u_{1}\left(t, x-\frac{b^{*} t}{\varepsilon}, \frac{x}{\varepsilon}\right) \\
& v_{\varepsilon}(t, x) \approx K u_{0}\left(t, x-\frac{b^{*} t}{\varepsilon}\right)+\varepsilon v_{1}\left(t, x-\frac{b^{*} t}{\varepsilon}, \frac{x}{\varepsilon}\right)
\end{aligned}
$$

with the effective drift

$$
b^{*}=\frac{\int_{Y^{0}} b(y) d y+K \int_{\partial \Sigma^{0}} b^{S}(y) d \sigma(y)}{\left|Y^{0}\right|+K\left|\partial \Sigma^{0}\right|_{d-1}}
$$


and $u_{0}$ the solution of the homogenized problem

$$
\left\{\begin{array}{l}
K_{d} \frac{\partial u_{0}}{\partial t}-\operatorname{div}_{x}\left(A^{*} \nabla_{x} u_{0}\right)=0 \text { in }(0, T) \times \mathbb{R}^{d} \\
K_{d} u_{0}(0, x)=\left|Y^{0}\right| u^{0}(x)+\left|\partial \Sigma^{0}\right|_{d-1} v^{0}(x), \quad x \in \mathbb{R}^{d}
\end{array}\right.
$$

where $K_{d}=\left|Y^{0}\right|+K\left|\partial \Sigma^{0}\right|_{d-1}$ and the dispersion tensor $A^{*}$ is given by

$$
\begin{gathered}
A_{i j}^{*}=\int_{Y^{0}} D(y)\left(\nabla_{y} \chi_{i}+e_{i}\right) \cdot\left(\nabla_{y} \chi_{j}+e_{j}\right) d y+\kappa \int_{\partial \Sigma^{0}}\left(\chi_{i}-\frac{\omega_{i}}{K}\right)\left(\chi_{j}-\frac{\omega_{j}}{K}\right) d \sigma(y) \\
\quad+K^{-1} \int_{\partial \Sigma^{0}} D^{S}(y)\left(K e_{i}+\nabla_{y}^{S} \omega_{i}\right) \cdot\left(K e_{j}+\nabla_{y}^{S} \omega_{j}\right) d \sigma(y)
\end{gathered}
$$

with $(\chi, \omega)=\left(\chi_{i}, \omega_{i}\right)_{1 \leq i \leq d}$ being the solution of the cell problem such that

$$
u_{1}(t, x, y)=\chi(y) \cdot \nabla_{x} u_{0}(t, x), \quad v_{1}(t, x, y)=\omega(y) \cdot \nabla_{x} u_{0}(t, x),
$$

and the cell problem is

$$
\begin{cases}b(y) \cdot \nabla_{y} \chi_{i}-\operatorname{div}_{y}\left(D\left(\nabla_{y} \chi_{i}+e_{i}\right)\right)=\left(b^{*}-b\right) \cdot e_{i} & \text { in } Y^{0}, \\ b^{S}(y) \cdot \nabla_{y}^{S} \omega_{i}-\operatorname{div}_{y}^{S}\left(D^{S}\left(\nabla_{y}^{S} \omega_{i}+K e_{i}\right)\right)=K\left(b^{*}-b^{S}\right) \cdot e_{i}+\kappa\left(\chi_{i}-K^{-1} \omega_{i}\right) & \text { on } \partial \Sigma^{0} \\ -D\left(\nabla_{y} \chi_{i}+e_{i}\right) \cdot n=\kappa\left(\chi_{i}-K^{-1} \omega_{i}\right) & \text { on } \partial \Sigma^{0} \\ y \rightarrow\left(\chi_{i}, \omega_{i}\right) & Y-\text { periodic. }\end{cases}
$$

Remark 4.4 In Proposition 4.3, the asymptotic profile $u_{0}$ is defined in a moving frame of reference. Of course, one can go back to a fixed frame of reference by defining

$$
\tilde{u}_{0}(t, x)=u_{0}\left(t, x-\frac{b^{*} t}{\varepsilon}\right) .
$$

Then, while $u_{0}$ was solution of the diffusion homogenized equation (14), $\tilde{u}_{0}$ is now solution of an homogenized convection-diffusion equation

$$
K_{d} \frac{\partial \tilde{u}_{0}}{\partial t}+\frac{1}{\varepsilon} b^{*} \cdot \nabla_{x} \tilde{u}_{0}-\operatorname{div}_{x}\left(A^{*} \nabla_{x} \tilde{u}_{0}\right)=0 \text { in }(0, T) \times \mathbb{R}^{d}
$$

which has a nicer physical interpretation since $b^{*} / \varepsilon$ is clearly the homogenized velocity.

Proof. Inserting the ansatz (8) and (9) in the equations (1) and (2) yields the following cascade of equations.

$$
\left\{\begin{array}{cl}
b(y) \cdot \nabla_{y} u_{0}-\operatorname{div}_{y}\left(D(y) \nabla_{y} u_{0}\right)=0 & \text { in } Y^{0} \\
-D \nabla_{y} u_{0} \cdot n=b^{S}(y) \cdot \nabla_{y}^{S} v_{0}-\operatorname{div}_{y}^{S}\left(D^{S}(y) \nabla_{y}^{S} v_{0}\right) & \\
=\kappa\left(u_{0}-K^{-1} v_{0}\right) & \text { on } \partial \Sigma^{0} \\
y \rightarrow\left(u_{0}(y), v_{0}(y)\right) & Y-\text { periodic. }
\end{array}\right.
$$

From Lemma 4.1 we deduce that the solution of (18) does not depend on $y$ and satisfy $v_{0}(t, x)=K u_{0}(t, x)$. 
At order $\varepsilon^{-1}$ :

$$
\left\{\begin{array}{cc}
-b^{*} \cdot \nabla_{x} u_{0}+b(y) \cdot\left(\nabla_{x} u_{0}+\nabla_{y} u_{1}\right)-\operatorname{div}_{y}\left(D(y)\left(\nabla_{x} u_{0}+\nabla_{y} u_{1}\right)\right)=0 & \text { in } Y^{0}, \\
-b^{*} \cdot \nabla_{x} v_{0}+b^{S}(y) \cdot\left(\nabla_{x}^{S} v_{0}+\nabla_{y}^{S} v_{1}\right)-\operatorname{div}_{y}^{S}\left(D^{S}(y)\left(\nabla_{x}^{S} v_{0}+\nabla_{y}^{S} v_{1}\right)\right) & \\
=-D(y)\left(\nabla_{x} u_{0}+\nabla_{y} u_{1}\right) \cdot n=\kappa\left(u_{1}-K^{-1} v_{1}\right) & \text { on } \partial \Sigma^{0}, \\
y \rightarrow\left(u_{1}(y), v_{1}(y)\right) & Y \text { - periodic. }
\end{array}\right.
$$

The compatibility condition (12) of Lemma 4.1 yields the desired value (13) of the drift velocity $b^{*}$ in order to solve (19). By linearity of (19) we deduce that its solution is given by

$$
u_{1}(t, x, y)=\chi(y) \cdot \nabla_{x} u_{0} \text { and } v_{1}(t, x, y)=\omega(y) \cdot \nabla_{x} u_{0}
$$

where $(\chi, \omega)$ is the solution of the cell problem (17).

At order $\varepsilon^{0}$ :

$$
\begin{cases}\frac{\partial}{\partial t} u_{0}-b^{*} \cdot \nabla_{x} u_{1}+b(y) \cdot\left(\nabla_{x} u_{1}+\nabla_{y} u_{2}\right) & \\ -\operatorname{div}_{x}\left(D(y)\left(\nabla_{x} u_{0}+\nabla_{y} u_{1}\right)\right)-\operatorname{div}_{y}\left(D(y)\left(\nabla_{x} u_{1}+\nabla_{y} u_{2}\right)\right)=0 & \text { in } Y^{0}, \\ \frac{\partial}{\partial t} v_{0}-b^{*} \cdot \nabla_{x} v_{1}+b^{S}(y) \cdot\left(\nabla_{x}^{S} u_{1}+\nabla_{y}^{S} u_{2}\right) & \\ -\operatorname{div}_{x}\left(D^{S}(y)\left(\nabla_{x} v_{0}+\nabla_{y}^{S} v_{1}\right)\right)-\operatorname{div}_{y}^{S}\left(D^{S}(y)\left(\nabla_{x} v_{1}+\nabla_{y}^{S} v_{2}\right)\right) & \\ =-D(y)\left(\nabla_{y} u_{2}+\nabla_{x} u_{1}\right) \cdot n=\kappa\left(u_{2}-K^{-1} v_{2}\right) & \text { on } \partial \Sigma^{0}, \\ y \rightarrow\left(u_{2}(y), v_{2}(y)\right) & Y \text { - periodic. }\end{cases}
$$

On identifying (20) with (11) we get the following right hand sides

$$
\begin{cases}f=\left(b^{*}-b\right) \cdot \nabla_{x} u_{1}+\operatorname{div}_{x}\left(D\left(\nabla_{x} u_{0}+\nabla_{y} u_{1}\right)\right)-\frac{\partial u_{0}}{\partial t}+\operatorname{div}_{y}\left(D\left(\nabla_{x} u_{1}\right)\right) & \text { in } Y^{0}, \\ g=-D \nabla_{x} u_{1} \cdot n & \text { on } \partial \Sigma^{0}, \\ h=-\frac{\partial v_{0}}{\partial t}+b^{*} \cdot \nabla_{x} v_{1}-b^{S} \cdot\left(\nabla_{x} u_{1}\right) & \\ \quad+\operatorname{div}_{x}\left(D^{S}\left(\nabla_{x} v_{0}+\nabla_{y}^{S} v_{1}\right)\right)+\operatorname{div}_{y}^{S}\left(D^{S} \nabla_{x} v_{1}\right) & \text { on } \partial \Sigma^{0} .\end{cases}
$$

According to Lemma 4.1, there exists a solution $\left(u_{2}, v_{2}\right)$ provided (12) holds true. This compatibility condition leads to the homogenized problem

$$
K_{d} \partial_{t} u_{0}=\operatorname{div}_{x}\left(A^{*} \nabla_{x} u_{0}\right) \text { in }(0, T) \times \mathbb{R}^{d}
$$

where $K_{d}=\left|Y^{0}\right|+K\left|\partial \Sigma^{0}\right|_{d-1}$ and the entries of the dispersion tensor $A^{*}$ are given by

$$
\begin{gathered}
A_{i j}^{*}=\int_{Y^{0}} D e_{i} \cdot e_{j} d y+\frac{1}{2}\left[\int_{Y^{0}} D \nabla_{y} \chi_{j} \cdot e_{i} d y+\int_{Y^{0}} D \nabla_{y} \chi_{i} \cdot e_{j} d y\right] \\
+K \int_{\partial \Sigma^{0}} D^{S} e_{i} \cdot e_{j} d \sigma(y)+\frac{1}{2}\left[\int_{\partial \Sigma^{0}} D^{S} \nabla_{y}^{S} \omega_{j} \cdot e_{i} d \sigma(y)+\int_{\partial \Sigma^{0}} D^{S} \nabla_{y}^{S} \omega_{i} \cdot e_{j} d \sigma(y)\right] \\
+\frac{1}{2}\left[\int_{Y^{0}}\left(b_{i}^{*}-b_{i}(y)\right) \chi_{j}(y) d y+\int_{Y^{0}}\left(b_{j}^{*}-b_{j}(y)\right) \chi_{i}(y) d y\right] \\
+\frac{1}{2}\left[\int_{\partial \Sigma^{0}}\left(b_{i}^{*}-b_{i}^{S}(y)\right) \omega_{j}(y) d \sigma(y)+\int_{\partial \Sigma^{0}}\left(b_{j}^{*}-b_{j}^{S}(y)\right) \omega_{i}(y) d \sigma(y)\right]
\end{gathered}
$$


Remark that we have symmetrized the dispersion tensor $A^{*}$ since only its contraction with the (symmetric) hessian matrix $\nabla^{2} u_{0}$ plays a role in the homogenized equation. In other words its antisymmetric part (if any) cannot be deduced from the above method of obtaining the homogenized equation. Testing the cell problem (17) for $\left(\chi_{i}, \omega_{i}\right)$ by $\left(\chi_{j}, \omega_{j}\right)$ and vice-versa leads to the following relationship

$$
\begin{aligned}
& \int_{Y^{0}} D \nabla_{y} \chi_{i} \cdot \nabla_{y} \chi_{j} d y+\frac{1}{K} \int_{\partial \Sigma^{0}} D^{S} \nabla_{y}^{S} \omega_{i} \cdot \nabla_{y}^{S} \omega_{j} d \sigma(y)+\kappa \int_{\partial \Sigma^{0}}\left[\chi_{i}-\frac{\omega_{i}}{K}\right]\left[\chi_{j}-\frac{\omega_{j}}{K}\right] d \sigma(y) \\
&+\frac{1}{2}\left[\int_{Y^{0}} D \nabla_{y} \chi_{j} \cdot e_{i} d y+\int_{Y^{0}} D \nabla_{y} \chi_{i} \cdot e_{j} d y\right] \\
&+ \frac{1}{2}\left[\int_{\partial \Sigma^{0}} D^{S} \nabla_{y}^{S} \omega_{j} \cdot e_{i} d \sigma(y)+\int_{\partial \Sigma^{0}} D^{S} \nabla_{y}^{S} \omega_{i} \cdot e_{j} d \sigma(y)\right] \\
&= \frac{1}{2}\left[\int_{Y^{0}}\left(b_{i}^{*}-b_{i}(y)\right) \chi_{j}(y) d y+\int_{Y^{0}}\left(b_{j}^{*}-b_{j}(y)\right) \chi_{i}(y) d y\right] \\
&+\frac{1}{2}\left[\int_{\partial \Sigma^{0}}\left(b_{i}^{*}-b_{i}^{S}(y)\right) \omega_{j}(y) d \sigma(y)+\int_{\partial \Sigma^{0}}\left(b_{j}^{*}-b_{j}^{S}(y)\right) \omega_{i}(y) d \sigma(y)\right]
\end{aligned}
$$

Adding the above equality to the previous expression for $A^{*}$ yields the desired formula (15). To obtain the initial condition of the homogenized equation we use a conservation property in the unit cell which says that

$$
\int_{Y^{0}} u_{0}(0, x) d y+\int_{\partial \Sigma^{0}} v_{0}(0, x) d y=\int_{Y^{0}} u^{0}(x) d y+\int_{\partial \Sigma^{0}} v^{0}(x) d y
$$

which leads to the desired initial condition upon recalling that $v_{0}=K u_{0}$. Let us emphasize once again that this proof is purely formal since it is based on the assumption (8)-(9) of a given ansatz for the solution $\left(u_{\varepsilon}, v_{\varepsilon}\right)$ (and we don't prove that such a series is converging).

\section{Two scale convergence with drift}

The goal of this section is to rigorously justify the homogenized limit heuristically established in the previous section. We shall use the notion of two-scale convergence with drift introduced in [21] (see [3] for detailed proofs). It is a generalization of the usual two-scale convergence defined in [2], [24].

Proposition 5.1 [21] Let $\mathcal{V}$ be a constant vector in $\mathbb{R}^{d}$. For any bounded sequence of functions $U_{\varepsilon}(t, x) \in L^{2}\left((0, T) \times \mathbb{R}^{d}\right)$, i.e., satisfying

$$
\left\|U_{\varepsilon}\right\|_{L^{2}\left((0, T) \times \mathbb{R}^{d}\right)} \leq C
$$

there exists a limit $U_{0}(t, x, y) \in L^{2}\left((0, T) \times \mathbb{R}^{d} \times \mathbb{T}^{d}\right)$ and one can extract a subsequence (still denoted by $\varepsilon$ ) which is said to two-scale converge with drift $\mathcal{V}$, or 
equivalently in moving coordinates $(t, x) \rightarrow\left(t, x-\frac{\mathcal{V} t}{\varepsilon}\right)$, to this limit, in the sense that, for any $\phi(t, x, y) \in C_{0}^{\infty}\left((0, T) \times \mathbb{R}^{d} \times \mathbb{T}^{d}\right)$,

$$
\lim _{\varepsilon \rightarrow 0} \int_{0}^{T} \int_{\mathbb{R}^{d}} U_{\varepsilon}(t, x) \phi\left(t, x-\frac{\mathcal{V} t}{\varepsilon}, \frac{x}{\varepsilon}\right) d x d t=\int_{0}^{T} \int_{\mathbb{R}^{d}} \int_{\mathbb{T}^{d}} U_{0}(t, x, y) \phi(t, x, y) d y d x d t .
$$

We denote this convergence by $U_{\varepsilon} \stackrel{2-\text { drift }}{\longrightarrow} U_{0}$.

Remark 5.2 Proposition 5.1 equally applies to a sequence $u_{\varepsilon}(t, x) \in L^{2}((0, T) \times$ $\left.\Omega_{\varepsilon}\right)$, merely defined in the perforated domain $\Omega_{\varepsilon}$, and satisfying the uniform bound

$$
\left\|u_{\varepsilon}\right\|_{L^{2}\left((0, T) \times \Omega_{\varepsilon}\right)} \leq C .
$$

Indeed, defining an extended function $U_{\varepsilon}(t, x)=u_{\varepsilon}(t, x)$ in $\Omega_{\varepsilon}$ and $U_{\varepsilon}(t, x)=0$ in $\mathbb{R}^{d} \backslash \Omega_{\varepsilon}$, we obtain that

$$
\int_{0}^{T} \int_{\Omega_{\varepsilon}} u_{\varepsilon}(t, x) \phi\left(t, x-\frac{\mathcal{V} t}{\varepsilon}, \frac{x}{\varepsilon}\right) d x d t=\int_{0}^{T} \int_{\mathbb{R}^{d}} U_{\varepsilon}(t, x) \phi\left(t, x-\frac{\mathcal{V} t}{\varepsilon}, \frac{x}{\varepsilon}\right) d x d t
$$

and the two-scale limit $U_{0}(t, x, y)$ of $U_{\varepsilon}$ vanishes in $\Sigma^{0}$ so that

$$
\lim _{\varepsilon \rightarrow 0} \int_{0}^{T} \int_{\Omega_{\varepsilon}} u_{\varepsilon}(t, x) \phi\left(t, x-\frac{\mathcal{V} t}{\varepsilon}, \frac{x}{\varepsilon}\right) d x d t=\int_{0}^{T} \int_{\mathbb{R}^{d}} \int_{Y^{0}} U_{0}(t, x, y) \phi(t, x, y) d y d x d t .
$$

Proposition 5.1 can be generalized in several ways as follows.

Proposition 5.3 Let $\mathcal{V}$ be a constant vector in $\mathbb{R}^{d}$ and let the sequence $U_{\varepsilon}$ be uniformly bounded in $L^{2}\left((0, T) ; H^{1}\left(\mathbb{R}^{d}\right)\right)$. Then, there exists a subsequence, still denoted by $\varepsilon$, and functions $U_{0}(t, x) \in L^{2}\left((0, T) ; H^{1}\left(\mathbb{R}^{d}\right)\right)$ and $U_{1}(t, x, y) \in L^{2}((0, T) \times$ $\left.\mathbb{R}^{d} ; H^{1}\left(\mathbb{T}^{d}\right)\right)$ such that

$$
U_{\varepsilon} \stackrel{2-d r i f t}{\longrightarrow} U_{0}
$$

and

$$
\nabla U_{\varepsilon} \stackrel{2-\text { drift }}{\longrightarrow} \nabla_{x} U_{0}+\nabla_{y} U_{1}
$$

The proof of Proposition 5.3 is a combination of arguments from [21], [3] and of the classical case without drift in [2], [24]. Two-scale convergence can also be defined for sequences defined on a periodic surface (see [5], [25] for the case without drift).

Proposition 5.4 Let $\mathcal{V}$ be a constant vector in $\mathbb{R}^{d}$ and let $W_{\varepsilon}$ be a sequence in $L^{2}\left((0, T) \times \partial \Omega_{\varepsilon}\right)$ such that

$$
\varepsilon \int_{0}^{T} \int_{\partial \Omega_{\varepsilon}}\left|W_{\varepsilon}(t, x)\right|^{2} d \sigma_{\varepsilon}(x) d t \leq C .
$$

Then, there exists a subsequence, still denoted by $\varepsilon$, and a function $W_{0}(t, x, y) \in$ $L^{2}\left((0, T) \times \mathbb{R}^{d} \times \partial \Sigma^{0}\right)$ such that $W_{\varepsilon}(t, x)$ two-scale converges with drift $\mathcal{V}$ to $W_{0}(t, x, y)$ in the sense that

$\lim _{\varepsilon \rightarrow 0} \varepsilon \int_{0}^{T} \int_{\partial \Omega_{\varepsilon}} W_{\varepsilon}(t, x) \phi\left(t, x-\frac{\mathcal{V} t}{\varepsilon}, \frac{x}{\varepsilon}\right) d \sigma_{\varepsilon}(x) d t=\int_{0}^{T} \int_{\mathbb{R}^{d}} \int_{\partial \Sigma^{0}} W_{0}(t, x, y) \phi(t, x, y) d \sigma(y) d x d t$

for any $\phi(t, x, y) \in C_{0}^{\infty}\left((0, T) \times \mathbb{R}^{d} \times \mathbb{T}^{d}\right)$. We denote this convergence by $W_{\varepsilon} \stackrel{2 S-\text { drift }}{\longrightarrow}$ $W_{0}$. 
Proposition 5.5 Let $W_{\varepsilon}(t, x) \in L^{2}\left((0, T) ; H^{1}\left(\partial \Omega_{\varepsilon}\right)\right)$ be such that

$$
\varepsilon \int_{0}^{T} \int_{\partial \Omega_{\varepsilon}}\left(\left|W_{\varepsilon}(t, x)\right|^{2}+\left|\nabla^{S} W_{\varepsilon}(t, x)\right|^{2}\right) d \sigma_{\varepsilon}(x) d t \leq C .
$$

There exist $W_{0}(t, x) \in L^{2}\left((0, T) ; H^{1}\left(\mathbb{R}^{d}\right)\right)$ and $W_{1}(t, x, y) \in L^{2}\left((0, T) \times \mathbb{R}^{d} ; H_{\#}^{1}\left(\partial \Sigma^{0}\right)\right)$ such that

$$
\begin{gathered}
W_{\varepsilon} \stackrel{2 S-d r i f t}{\longrightarrow} W_{0}(t, x) \\
\nabla^{S} W_{\varepsilon} \stackrel{2 S-d r i f t}{\longrightarrow} G(y) \nabla_{x} W_{0}(t, x)+\nabla_{y}^{S} W_{1}(t, x, y)
\end{gathered}
$$

where $G(y)$ is the projection operator on the tangent plane of $\partial \Sigma^{0}$ at the point $y$.

The proofs of Propositions 5.4 and 5.5 are a slight modification of those in the classical case without drift which are to be found in [5], [25].

Eventually we state a technical lemma which will play a key role in the convergence analysis.

Lemma 5.6 Let $\phi(t, x, y) \in L^{2}\left((0, T) \times \mathbb{R}^{d} \times \partial \Sigma^{0}\right)$ be such that $\int_{\partial \Sigma^{0}} \phi(t, x, y) d \sigma(y)=$ 0 for every $(t, x) \in(0, T) \times \mathbb{R}^{d}$. There exist two vector fields $\theta(t, x, y) \in\left[L^{2}((0, T) \times\right.$ $\left.\left.\mathbb{R}^{d} \times \mathbb{T}^{d}\right)\right]^{d}$ and $\Theta(t, x, y) \in\left[L^{2}\left((0, T) \times \mathbb{R}^{d} \times \partial \Sigma^{0}\right)\right]^{d}$ such that

$$
\begin{array}{ll}
\operatorname{div}_{y} \theta=0 & \text { in } Y^{0}, \\
\theta \cdot n=\phi & \text { on } \partial \Sigma^{0}, \\
\operatorname{div}_{y}^{S} \Theta=\phi & \text { on } \partial \Sigma^{0} .
\end{array}
$$

Proof. We choose $\theta=\nabla_{y} \xi$ with $\xi \in H_{\#}^{1}\left(Y^{0}\right)$ a solution to

$$
\begin{cases}\Delta_{y} \xi=0 & \text { in } Y^{0} \\ \nabla_{y} \xi \cdot n=\phi & \text { on } \partial \Sigma^{0}\end{cases}
$$

which admits a unique solution, up to an additive constant, since the compatibility condition of (23) is satisfied. On similar lines, we choose $\Theta=\nabla_{y}^{S} \beta$ where $\beta$ is the unique solution in $H_{\#}^{1}\left(\partial \Sigma^{0}\right) / \mathbb{R}$ of $\Delta_{y}^{S} \beta=\phi$ on $\partial \Sigma^{0}$ which is solvable because $\int_{\partial \Sigma^{0}} \phi d \sigma(y)=0$

We now apply the above results on two-scale convergence with drift to the homogenization of (1)-(3). Let us choose the drift $\mathcal{V}=b^{*}$ given by formula (13).

Theorem 5.7 The sequence of bulk and surface concentrations $\left\{u_{\varepsilon}\right\}$ and $\left\{v_{\varepsilon}\right\}$, solutions of system (1)-(3), two-scale converges with drift $\mathcal{V}$, as $\varepsilon \rightarrow 0$, in the following sense

$$
\left\{\begin{array}{l}
u_{\varepsilon} \stackrel{2-\text { drift }}{\text { }} u_{0}(t, x) \\
v_{\varepsilon} \stackrel{2 S-\text { drift }}{T} K u_{0}(t, x) \\
\nabla u_{\varepsilon} \stackrel{2-\text { drift }}{\longrightarrow} \nabla_{x} u_{0}(t, x)+\nabla_{y}\left(\chi(y) \cdot \nabla_{x} u_{0}(t, x)\right) \\
\nabla^{S} v_{\varepsilon} \stackrel{2 S-\text { drift }}{\longrightarrow} K G(y) \nabla_{x} u_{0}(t, x)+\nabla_{y}^{S} \omega(y) \cdot \nabla_{x} u_{0}(t, x) \\
\frac{1}{\varepsilon}\left(u_{\varepsilon}-\frac{1}{K} v_{\varepsilon}\right) \stackrel{2 S-d r i f t}{\longrightarrow}(\chi(y)-\omega(y)) \cdot \nabla_{x} u_{0}(t, x)
\end{array}\right.
$$

where $u_{0}(t, x)$ is the unique solution of the homogenized problem (14) and $(\chi(y), \omega(y))=$ $\left(\chi_{i}(y), \omega_{i}(y)\right)_{1 \leq i \leq d}$ are the solutions of the cell problem (17). 
Proof. Up to extracting a subsequence, the two scale convergence results (24) are obvious consequences of the a priori estimates of Lemma 3.1 and of the previous Propositions 5.3 and 5.5 (see [6] for similar computations, if necessary). The only limit that deserves some attention is that for $w_{\varepsilon}=\frac{1}{\varepsilon}\left(u_{\varepsilon}-K^{-1} v_{\varepsilon}\right)$. From Lemma 3.1 we know that it satisfies the uniform estimate

$$
\varepsilon \int_{0}^{T} \int_{\partial \Omega_{\varepsilon}}\left|w_{\varepsilon}(t, x)\right|^{2} d \sigma_{\varepsilon}(x) d t \leq C,
$$

from which, by virtue of Proposition 5.4, we deduce, for a subsequence, that $w_{\varepsilon} \stackrel{2 S-\text { drift }}{\longrightarrow} q(t, x, y)$ for some $q(t, x, y) \in L^{2}\left((0, T) \times \mathbb{R}^{d} ; L^{2}\left(\partial \Sigma^{0}\right)\right)$. Let us choose a test function $\phi$ as in Lemma 5.6, i.e., $\int_{\partial \Sigma^{0}} \phi(t, x, y) d \sigma(y)=0$. To pass to the limit in

$$
\lim _{\varepsilon \rightarrow 0} \varepsilon \int_{0}^{T} \int_{\partial \Omega_{\varepsilon}} w_{\varepsilon}(t, x) \phi\left(t, x-\frac{b^{*} t}{\varepsilon}, \frac{x}{\varepsilon}\right) d \sigma_{\varepsilon}(x) d t
$$

we separate $w_{\varepsilon}$ in a difference of two terms. In view of (22), the first one is

$$
\begin{gathered}
\varepsilon \int_{0}^{T} \int_{\partial \Omega_{\varepsilon}} \frac{1}{\varepsilon} u_{\varepsilon} \phi\left(t, x-\frac{b^{*} t}{\varepsilon}, \frac{x}{\varepsilon}\right) d \sigma_{\varepsilon}(x) d t=\int_{0}^{T} \int_{\Omega_{\varepsilon}} \operatorname{div}\left(u_{\varepsilon} \theta\left(t, x-\frac{b^{*} t}{\varepsilon}, \frac{x}{\varepsilon}\right)\right) d x d t \\
=\int_{0}^{T} \int_{\Omega_{\varepsilon}}\left[\nabla u_{\varepsilon} \cdot \theta\left(t, x-\frac{b^{*} t}{\varepsilon}, \frac{x}{\varepsilon}\right)+u_{\varepsilon}\left(\operatorname{div}_{x} \theta\right)\left(t, x-\frac{b^{*} t}{\varepsilon}, \frac{x}{\varepsilon}\right)\right] d x d t \\
\frac{2 S-d r i f t}{\int_{0}^{T}} \int_{\mathbb{R}^{d}} \int_{Y^{0}}\left[\left(\nabla_{x} u+\nabla_{y} u_{1}\right) \cdot \theta+u \operatorname{div}_{x} \theta\right] d y d x d t \\
=\int_{0}^{T} \int_{\mathbb{R}^{d}} \int_{\partial \Sigma^{0}} u_{1} \theta \cdot n d \sigma(y) d x d t=\int_{0}^{T} \int_{\mathbb{R}^{d}} \int_{\partial \Sigma^{0}} u_{1} \phi d \sigma(y) d x d t .
\end{gathered}
$$

Now, the second term is

$$
\begin{aligned}
& \varepsilon \int_{0}^{T} \int_{\partial \Omega_{\varepsilon}} \frac{1}{K \varepsilon} v_{\varepsilon} \phi\left(t, x-\frac{b^{*} t}{\varepsilon}, \frac{x}{\varepsilon}\right) d \sigma_{\varepsilon}(x) d t=\int_{0}^{T} \int_{\partial \Omega_{\varepsilon}} \frac{1}{K} v_{\varepsilon}\left(\operatorname{div}_{y}^{S} \Theta\right)\left(t, x-\frac{b^{*} t}{\varepsilon}, \frac{x}{\varepsilon}\right) d \sigma_{\varepsilon}(x) d t \\
& =\varepsilon \int_{0}^{T} \int_{\partial \Omega_{\varepsilon}} \frac{1}{K} v_{\varepsilon}\left[\operatorname{div}^{S}\left(\Theta\left(t, x-\frac{b^{*} t}{\varepsilon}, \frac{x}{\varepsilon}\right)\right)-\operatorname{div}_{x}(G \Theta)\left(t, x-\frac{b^{*} t}{\varepsilon}, \frac{x}{\varepsilon}\right)\right] d \sigma_{\varepsilon}(x) d t \\
& =\varepsilon \int_{0}^{T} \int_{\partial \Omega_{\varepsilon}} \frac{1}{K}\left[-\Theta\left(t, x-\frac{b^{*} t}{\varepsilon}, \frac{x}{\varepsilon}\right) \cdot \nabla^{S} v_{\varepsilon}-\operatorname{div}_{x}(G \Theta)\left(t, x-\frac{b^{*} t}{\varepsilon}, \frac{x}{\varepsilon}\right) v_{\varepsilon}\right] d \sigma_{\varepsilon}(x) d t \\
& \underline{2 S-d r i f t} \int_{0}^{T} \int_{\mathbb{R}^{d}} \int_{\partial \Sigma^{0}}^{T} \frac{1}{K}\left[-\Theta \cdot\left(K G(y) \nabla_{x} u_{0}+\nabla_{y}^{S} v_{1}\right)-\operatorname{div}_{x}(G(y) \Theta) K u_{0}\right] d \sigma(y) d x d t \\
& =\int_{0}^{T} \int_{\mathbb{R}^{d}} \int_{\partial \Sigma^{0}} \frac{1}{K} v_{1} \operatorname{div}_{y}^{S} \Theta d \sigma(y) d x d t=\int_{0}^{T} \int_{\mathbb{R}^{d}} \int_{\partial \Sigma^{0}} \frac{1}{K} v_{1} \phi d \sigma(y) d x d t
\end{aligned}
$$


Subtracting the two terms, we have shown that

$$
\int_{0}^{T} \int_{\mathbb{R}^{d}} \int_{\partial \Sigma^{0}} q \phi d \sigma(y) d x d t=\int_{0}^{T} \int_{\mathbb{R}^{d}} \int_{\partial \Sigma^{0}}\left(u_{1}-\frac{v_{1}}{K}\right) \phi d \sigma(y) d x d t
$$

for all $\phi$ such that $\int_{\partial \Sigma^{0}} \phi d y=0$. Thus,

$$
q(t, x, y)=u_{1}(t, x, y)-\frac{v_{1}(t, x, y)}{K}+l(t, x)
$$

for some function $l(t, x)$ which does not depend on $y$. Since, $u_{1}$ and $v_{1}$ are also defined up to the addition of a function solely dependent on $(t, x)$, we can get rid of $l(t, x)$ and we recover indeed the last line of $(24)$.

The rest of the proof is now devoted to show that $u_{0}(t, x)$ is the solution of the homogenized equation (14). For that goal, we shall pass to the limit in the coupled variational formulation of (1)-(3),

$$
\begin{aligned}
& \int_{0}^{T} \int_{\Omega_{\varepsilon}}\left[\frac{\partial u_{\varepsilon}}{\partial t} \phi_{\varepsilon}+\frac{1}{\varepsilon} b_{\varepsilon} \cdot \nabla u_{\varepsilon} \phi_{\varepsilon}+D_{\varepsilon} \nabla u_{\varepsilon} \cdot \nabla \phi_{\varepsilon}\right] d x d t \\
+ & \int_{0}^{T} \int_{\partial \Omega_{\varepsilon}} \frac{1}{K}\left[\frac{\partial v_{\varepsilon}}{\partial t} \psi_{\varepsilon}+\frac{1}{\varepsilon} b_{\varepsilon}^{S} \cdot \nabla^{S} v_{\varepsilon} \psi_{\varepsilon}+D_{\varepsilon}^{S} \nabla^{S} v_{\varepsilon} \cdot \nabla^{S} \psi_{\varepsilon}\right] d \sigma_{\varepsilon}(x) d t \\
& +\int_{0}^{T} \int_{\partial \Omega_{\varepsilon}}\left[\frac{\kappa}{\varepsilon}\left(u_{\varepsilon}-\frac{v_{\varepsilon}}{K}\right)\left(\phi_{\varepsilon}-\frac{\psi_{\varepsilon}}{K}\right)\right] d \sigma_{\varepsilon}(x) d t=0
\end{aligned}
$$

with the test functions

$$
\begin{gathered}
\phi_{\varepsilon}=\phi\left(t, x-\frac{b^{*} t}{\varepsilon}\right)+\varepsilon \phi_{1}\left(t, x-\frac{b^{*} t}{\varepsilon}, \frac{x}{\varepsilon}\right), \\
\psi_{\varepsilon}=K \phi\left(t, x-\frac{b^{*} t}{\varepsilon}\right)+\varepsilon \psi_{1}\left(t, x-\frac{b^{*} t}{\varepsilon}, \frac{x}{\varepsilon}\right) .
\end{gathered}
$$

Here $\phi(t, x), \phi_{1}(t, x, y)$ and $\psi_{1}(t, x, y)$ are smooth compactly supported functions which vanish at $t=T$. Let us consider the convective terms in (25) and perform integrations by parts:

$$
\begin{aligned}
& \int_{0}^{T} \int_{\Omega_{\varepsilon}}\left(\frac{\partial u_{\varepsilon}}{\partial t}+\frac{1}{\varepsilon} b_{\varepsilon} \cdot \nabla u_{\varepsilon}\right) \phi_{\varepsilon} d x d t+\varepsilon \int_{0}^{T} \int_{\partial \Omega_{\varepsilon}} \frac{1}{K}\left(\frac{\partial v_{\varepsilon}}{\partial t}+\frac{1}{\varepsilon} b_{\varepsilon}^{S} \cdot \nabla^{S} v_{\varepsilon}\right) \psi_{\varepsilon} d \sigma_{\varepsilon}(x) d t \\
& =-\int_{0}^{T} \int_{\Omega_{\varepsilon}} u_{\varepsilon} \frac{\partial \phi}{\partial t}\left(t, x-\frac{b^{*} t}{\varepsilon}\right) d x d t+\frac{1}{\varepsilon} \int_{0}^{T} \int_{\Omega_{\varepsilon}} u_{\varepsilon} b^{*} \cdot \nabla_{x} \phi\left(t, x-\frac{b^{*} t}{\varepsilon}\right) d x d t \\
& \quad+\int_{0}^{T} \int_{\Omega_{\varepsilon}} u_{\varepsilon} b^{*} \cdot \nabla_{x} \phi_{1}\left(t, x-\frac{b^{*} t}{\varepsilon}, \frac{x}{\varepsilon}\right) d x d t-\int_{\Omega_{\varepsilon}} u^{0}(x) \phi(0, x) d x+O(\varepsilon) \\
& -\frac{1}{\varepsilon} \int_{0}^{T} \int_{\Omega_{\varepsilon}}^{T} u_{\varepsilon} b_{\varepsilon} \cdot \nabla_{x} \phi\left(t, x-\frac{b^{*} t}{\varepsilon}\right) d x d t+\int_{0}^{T} \int_{\Omega_{\varepsilon}} b_{\varepsilon} \cdot \nabla u_{\varepsilon} \phi_{1}\left(t, x-\frac{b^{*} t}{\varepsilon}, \frac{x}{\varepsilon}\right) d x d t
\end{aligned}
$$




$$
\begin{aligned}
& -\varepsilon \int_{0}^{T} \int_{\partial \Omega_{\varepsilon}} v_{\varepsilon} \frac{\partial \phi}{\partial t}\left(t, x-\frac{b^{*} t}{\varepsilon}\right) d \sigma_{\varepsilon}(x) d t+\int_{0}^{T} \int_{\partial \Omega_{\varepsilon}} v_{\varepsilon} b^{*} \cdot \nabla_{x} \phi\left(t, x-\frac{b^{*} t}{\varepsilon}\right) d \sigma_{\varepsilon}(x) d t \\
& +\frac{\varepsilon}{K} \int_{0}^{T} \int_{\partial \Omega_{\varepsilon}} v_{\varepsilon} b^{*} \cdot \nabla_{x} \psi_{1}\left(t, x-\frac{b^{*} t}{\varepsilon}, \frac{x}{\varepsilon}\right) d \sigma_{\varepsilon}(x) d t-\varepsilon \int_{\partial \Omega_{\varepsilon}} v^{0}(x) \phi(0, x) d \sigma_{\varepsilon}(x)+O(\varepsilon) \\
& -\int_{0}^{T} \int_{\partial \Omega_{\varepsilon}} v_{\varepsilon} b_{\varepsilon}^{S} \cdot \nabla_{x} \phi\left(t, x-\frac{b^{*} t}{\varepsilon}\right) d \sigma_{\varepsilon}(x) d t+\int_{0}^{T} \int_{\partial \Omega_{\varepsilon}} \frac{\varepsilon}{K} b_{\varepsilon}^{S} \cdot \nabla^{S} v_{\varepsilon} \psi_{1}\left(t, x-\frac{b^{*} t}{\varepsilon}, \frac{x}{\varepsilon}\right) d \sigma_{\varepsilon}(x) d t .
\end{aligned}
$$

We cannot directly pass to the two-scale limit since there are terms which apparently are of order $\varepsilon^{-1}$. We thus regroup them

$$
\begin{gathered}
\int_{0}^{T} \int_{\Omega_{\varepsilon}} u_{\varepsilon} \frac{b^{*}-b_{\varepsilon}}{\varepsilon} \cdot \nabla_{x} \phi\left(t, x-\frac{b^{*} t}{\varepsilon}\right) d x d t+\int_{0}^{T} \int_{\partial \Omega_{\varepsilon}} v_{\varepsilon}\left(b^{*}-b_{\varepsilon}^{S}\right) \cdot \nabla_{x} \phi\left(t, x-\frac{b^{*} t}{\varepsilon}\right) d \sigma_{\varepsilon}(x) d t \\
=\int_{0}^{T} \int_{\Omega_{\varepsilon}} u_{\varepsilon} \frac{b^{*}-b_{\varepsilon}}{\varepsilon} \cdot \nabla_{x} \phi\left(t, x-\frac{b^{*} t}{\varepsilon}\right) d x d t+K \int_{0}^{T} \int_{\partial \Omega_{\varepsilon}} u_{\varepsilon}\left(b^{*}-b_{\varepsilon}^{S}\right) \cdot \nabla_{x} \phi\left(t, x-\frac{b^{*} t}{\varepsilon}\right) d \sigma_{\varepsilon}(x) d t \\
+K \varepsilon \int_{0}^{T} \int_{\partial \Omega_{\varepsilon}} \frac{1}{\varepsilon}\left(u_{\varepsilon}-\frac{v_{\varepsilon}}{K}\right)\left(b_{\varepsilon}^{S}-b^{*}\right) \cdot \nabla_{x} \phi\left(t, x-\frac{b^{*} t}{\varepsilon}\right) d \sigma_{\varepsilon}(x) d t .
\end{gathered}
$$

We introduce an auxiliary problem

$$
\begin{cases}\Delta \alpha_{i}(y)=b_{i}(y)-b_{i}^{*} & \text { in } Y^{0} \\ \nabla \alpha_{i} \cdot n=K\left(b_{i}^{*}-b_{i}^{s}(y)\right) & \text { on } \partial \Sigma^{0} \\ \alpha_{i} & \text { is } Y \text { - periodic }\end{cases}
$$

which admits a unique solution (up to an additive constant) since, by definition of $b^{*}$, the source terms in (27) are in equilibrium. Defining $\alpha^{\varepsilon}(x)=\alpha(x / \varepsilon)$, thanks to (27), the $\varepsilon^{-1}$-order term (26) is equal to

$$
\begin{gathered}
\int_{0}^{T} \int_{\Omega_{\varepsilon}} \varepsilon \sum_{i=1}^{d} \nabla \alpha_{i}^{\varepsilon} \cdot \nabla\left(\partial_{x_{i}} \phi\left(t, x-\frac{b^{*} t}{\varepsilon}\right) u_{\varepsilon}\right) d x d t \\
+K \varepsilon \int_{0}^{T} \int_{\partial \Omega_{\varepsilon}} \frac{1}{\varepsilon}\left(u_{\varepsilon}-\frac{v_{\varepsilon}}{K}\right)\left(b_{\varepsilon}^{S}-b^{*}\right) \cdot \nabla_{x} \phi\left(t, x-\frac{b^{*} t}{\varepsilon}\right) d \sigma_{\varepsilon}(x) d t
\end{gathered}
$$

for which we can pass to the two-scale limit.

In a first step, we choose $\phi \equiv 0$, i.e., we consider only the terms involving $\phi_{1}$ and $\psi_{1}$ in the variational formulation (25)

$$
\begin{aligned}
& \int_{0}^{T} \int_{\Omega_{\varepsilon}} u_{\varepsilon} b^{*} \cdot \nabla_{x} \phi_{1}\left(t, x-\frac{b^{*} t}{\varepsilon}, \frac{x}{\varepsilon}\right) d x d t+\int_{0}^{T} \int_{\Omega_{\varepsilon}} b_{\varepsilon} \cdot \nabla u_{\varepsilon} \phi_{1}\left(t, x-\frac{b^{*} t}{\varepsilon}, \frac{x}{\varepsilon}\right) d x d t \\
& +\int_{0}^{T} \int_{\Omega_{\varepsilon}} D_{\varepsilon} \nabla u_{\varepsilon} \cdot \nabla_{y} \phi_{1}\left(t, x-\frac{b^{*} t}{\varepsilon}, \frac{x}{\varepsilon}\right) d x d t+\frac{\varepsilon}{K} \int_{0}^{T} \int_{\partial \Omega_{\varepsilon}} v_{\varepsilon} b^{*} \cdot \nabla_{x} \psi_{1}\left(t, x-\frac{b^{*} t}{\varepsilon}, \frac{x}{\varepsilon}\right) d \sigma_{\varepsilon}(x) d t
\end{aligned}
$$




$$
\begin{gathered}
+\int_{0}^{T} \int_{\partial \Omega_{\varepsilon}} \frac{\varepsilon}{K} b_{\varepsilon}^{S} \cdot \nabla^{S} v_{\varepsilon} \psi_{1}\left(t, x-\frac{b^{*} t}{\varepsilon}, \frac{x}{\varepsilon}\right) d \sigma_{\varepsilon}(x) d t+\frac{\varepsilon}{K} \int_{0}^{T} \int_{\partial \Omega_{\varepsilon}} D_{\varepsilon}^{S} \nabla^{S} v_{\varepsilon} \cdot \nabla_{y}^{S} \psi_{1}\left(t, x-\frac{b^{*} t}{\varepsilon}, \frac{x}{\varepsilon}\right) d \sigma_{\varepsilon}(x) d t \\
+\varepsilon \int_{0}^{T} \int_{\partial \Omega_{\varepsilon}} \frac{\kappa}{\varepsilon}\left(u_{\varepsilon}-\frac{v_{\varepsilon}}{K}\right)\left(\phi_{1}-\frac{\psi_{1}}{K}\right) d \sigma_{\varepsilon}(x) d t=0 .
\end{gathered}
$$

Passing to the two-scale limit with drift, we obtain

$$
\begin{gathered}
-\iint_{\mathbb{R}^{d}} b_{Y^{0}} \cdot \nabla_{x} u_{0}(t, x) \phi_{1}(t, x, y) d y d x d t+\iint_{\mathbb{R}^{d}} b(y) \cdot\left(\nabla_{x} u_{0}(t, x)+\nabla_{y} u_{1}(t, x, y)\right) \phi_{1}(t, x, y) d y d x d t \\
-\iint_{\mathbb{R}^{d}} \operatorname{div}_{Y^{0}}\left(D(y)\left(\nabla_{x} u_{0}(t, x)+\nabla_{y} u_{1}(t, x, y)\right)\right) \phi_{1}(t, x, y) d y d x d t \\
-\iint_{\mathbb{R}^{d}} \int_{\partial \Sigma^{0}} b^{*} \cdot \nabla_{x} u_{0}(t, x) \psi_{1}(t, x, y) d \sigma(y) d x d t \\
+\frac{1}{K} \int_{\mathbb{R}^{d}} \int_{\partial \Sigma^{0}} b^{S}(y) \cdot\left(K G \nabla_{x} u_{0}(t, x)+\nabla^{S} v_{1}(t, x, y)\right) \psi_{1}(t, x, y) d \sigma(y) d x d t \\
-\frac{1}{K} \int_{\mathbb{R}^{d}} \int_{\partial \Sigma^{0}} \operatorname{div}_{y}^{s}\left(D^{S}(y)\left(K G \nabla_{x} u_{0}(t, x)+\nabla^{S} v_{1}(t, x, y)\right)\right) \psi_{1}(t, x, y) d \sigma(y) d x d t \\
+\int_{\mathbb{R}^{d}} \int_{\partial \Sigma^{0}} \kappa\left(u_{1}-\frac{v_{1}}{K}\right)\left(\phi_{1}-\frac{\psi_{1}}{K}\right) d \sigma(y) d x d t=0
\end{gathered}
$$

The above expression is precisely the variational formulation of (19) which leads to the cell problem (17).

In a second step we choose $\phi_{1} \equiv 0$ and $\psi_{1} \equiv 0$ in (25), i.e., we consider only the terms involving $\phi$

$$
\begin{gathered}
-\int_{0}^{T} \int_{\Omega_{\varepsilon}} u_{\varepsilon} \frac{\partial \phi}{\partial t}\left(t, x-\frac{b^{*} t}{\varepsilon}\right) d x d t+\int_{0}^{T} \int_{\Omega_{\varepsilon}} D_{\varepsilon} \nabla u_{\varepsilon} \cdot \nabla_{x} \phi\left(t, x-\frac{b^{*} t}{\varepsilon}\right) d x d t \\
-\varepsilon \int_{0}^{T} \int_{\partial \Omega_{\varepsilon}} v_{\varepsilon} \frac{\partial \phi}{\partial t}\left(t, x-\frac{b^{*} t}{\varepsilon}\right) d \sigma_{\varepsilon}(x) d t+\varepsilon \int_{0}^{T} \int_{\partial \Omega_{\varepsilon}} D_{\varepsilon}^{S} \nabla^{S} v_{\varepsilon} \cdot \nabla^{S} \phi\left(t, x-\frac{b^{*} t}{\varepsilon}\right) d \sigma_{\varepsilon}(x) d t \\
+\int_{0}^{T} \int_{\Omega_{\varepsilon}} \varepsilon \sum_{i=1}^{d} \nabla \alpha_{i}^{\varepsilon} \cdot \nabla\left(\partial_{x_{i}} \phi\left(t, x-\frac{b^{*} t}{\varepsilon}\right) u_{\varepsilon}\right) d x d t \\
+K \varepsilon \int_{0}^{T} \int_{\partial \Omega_{\varepsilon}} \frac{1}{\varepsilon}\left(u_{\varepsilon}-\frac{v_{\varepsilon}}{K}\right)\left(b_{\varepsilon}^{S}-b^{*}\right) \cdot \nabla_{x} \phi\left(t, x-\frac{b^{*} t}{\varepsilon}\right) d \sigma_{\varepsilon}(x) d t \\
-\int_{\Omega_{\varepsilon}} u^{0}(x) \phi(0, x) d x-\varepsilon \int_{\partial \Omega_{\varepsilon}} v^{0}(x) \phi(0, x) d \sigma_{\varepsilon}(x)=0 .
\end{gathered}
$$

Taking into account formula (16) for $u_{1}$ and $v_{1}$, passing to the two-scale limit with drift yields

$$
\left|Y^{0}\right| \int_{\mathbb{R}^{d}} \frac{\partial u_{0}}{\partial t} \phi d x d t-\int_{\mathbb{R}^{d}} \int_{Y^{0}} \sum_{i, j=1}^{d} D_{i j}(y) \frac{\partial^{2} u}{\partial x_{i} \partial x_{j}} \phi d x d t
$$




$$
\begin{gathered}
-\left|Y^{0}\right| \int_{\mathbb{R}^{d}} u^{0}(x) \phi(0, x) d x-\int_{\mathbb{R}^{d}} \int_{Y^{0}} \sum_{i, j=1}^{d} \sum_{l=1}^{d} D_{i l}(y) \frac{\partial^{2} u_{0}}{\partial x_{i} \partial x_{j}} \frac{\partial \chi_{j}(y)}{\partial y_{l}} \phi d x d t \\
+K\left|\partial \Sigma^{0}\right| \int_{\mathbb{R}^{d}} \frac{\partial u_{0}}{\partial t} \phi d x d t-\int_{\mathbb{R}^{d}} \int_{\partial \Sigma^{0}} K \sum_{i, j=1}^{d} \sum_{l=1}^{d} D_{i l}^{S}(y) G_{l j}(y) \frac{\partial^{2} u_{0}}{\partial x_{i} \partial x_{j}} \phi d \sigma(y) d x d t \\
-K\left|\partial \Sigma^{0}\right| \int_{\mathbb{R}^{d}} u^{0}(x) \phi(0, x) d x-\iint_{\mathbb{R}^{d}} \int_{\Sigma^{0}} \sum_{i, j=1}^{d} \sum_{l=1}^{d} D_{i l}^{S}(y) \frac{\partial^{2} u_{0}}{\partial x_{i} \partial x_{j}} \frac{\partial^{S} \omega_{j}(y)}{\partial y_{l}} \phi d \sigma(y) d x d t \\
-K \int_{\mathbb{R}^{d}} \int_{\partial \Sigma^{0}} \sum_{i, j=1}^{d}\left(\chi_{j}-\frac{\omega_{j}}{K}\right)\left(b_{i}^{S}(y)-b_{i}^{*}\right) \frac{\partial^{2} u_{0}}{\partial x_{i} \partial x_{j}} \phi d \sigma(y) d x d t \\
-\int_{\mathbb{R}^{d}} \int_{Y^{0}} \sum_{i, j=1}^{d} \sum_{l=1}^{d} \frac{\partial \alpha_{i}(y)}{\partial y_{l}} \frac{\partial \chi_{j}(y)}{\partial y_{l}} \frac{\partial^{2} u_{0}}{\partial x_{i} \partial x_{j}} \phi d y d x d t=0 .
\end{gathered}
$$

Introducing $K_{d}=\left|Y^{0}\right|+K\left|\partial \Sigma^{0}\right|_{d-1}$, the above equation is just the variational formulation of the homogenized problem

$$
\left\{\begin{array}{l}
K_{d} \partial_{t} u_{0}=\operatorname{div}_{x}\left(A^{*} \nabla_{x} u_{0}\right) \text { in }(0, T) \times \mathbb{R}^{d} \\
K_{d} u_{0}(0, x)=\left|Y^{0}\right| u^{0}(x)+\left|\partial \Sigma^{0}\right|_{d-1} v^{0}(x), \quad x \in \mathbb{R}^{d}
\end{array}\right.
$$

The only difficulty is to recognize that the above cell average for $A^{*}$ coincides with formula (15). To check this point, one needs to test with $\chi_{j}$ the boundary value problem (27) for $\alpha$ which yields

$\int_{Y^{0}} \sum_{l=1}^{d} \frac{\partial \alpha_{i}(y)}{\partial y_{l}} \frac{\partial \chi_{j}(y)}{\partial y_{l}} d y=\int_{Y^{0}}\left(b_{i}^{*}-b_{i}(y)\right) \chi_{j}(y) d y+K \int_{\partial \Sigma^{0}}\left(b_{i}^{*}-b_{i}^{S}(y)\right) \chi_{j}(y) d \sigma(y)$.

We safely leave the other details of checking the formula for $A^{*}$ to the reader.

Finally, although we proved convergence only for a subsequence (following the statements of Propositions 5.3 and 5.5), the uniqueness of the homogenized solution $u_{0}(t, x)$ to $(28)$ implies that the entire sequence $\left\{u_{\varepsilon}, v_{\varepsilon}\right\}$ converge.

\section{Strong convergence}

In the previous section, we proved the (weak) two-scale convergence with drift of $\left(u_{\varepsilon}, v_{\varepsilon}\right)$ to $\left(u_{0}, K u_{0}\right)$. In this section, we improve Theorem 5.7 by proving that the convergence is actually strong, in a sense which is made precise in the following Theorem.

Theorem 6.1 Let $\left(u_{\varepsilon}, v_{\varepsilon}\right)$ be the solution to (1)-(3). Then, $u_{\varepsilon}(t, x) \mathbb{I}_{\Omega_{\varepsilon}}$ strongly two-scale converges with drift towards $\mathbb{I}_{Y 0} u_{0}(t, x)$ and $v_{\varepsilon}(t, x) \mathbb{I}_{\partial \Omega_{\varepsilon}}$ strongly twoscale converges with drift on surfaces towards $K \mathbb{I}_{\partial \Sigma^{0}} u_{0}(t, x)$, in the sense that

$$
\begin{aligned}
& \lim _{\varepsilon \rightarrow 0}\left\|u_{\varepsilon}(t, x)-u_{0}\left(t, x-\frac{b^{*}}{\varepsilon} t\right)\right\|_{L^{2}\left((0, T) \times \Omega_{\varepsilon}\right)}=0, \\
& \lim _{\varepsilon \rightarrow 0} \sqrt{\varepsilon}\left\|v_{\varepsilon}(t, x)-K u_{0}\left(t, x-\frac{b^{*}}{\varepsilon} t\right)\right\|_{L^{2}\left((0, T) \times \partial \Omega_{\varepsilon}\right)}=0 .
\end{aligned}
$$


Our proof of Theorem 6.1 is based on the following result about strong two-scale convergence (see Theorem 1.8 in [2] or Proposition 9.1 in [3]).

Proposition 6.2 Let $\left(U_{\varepsilon}\right)_{\varepsilon>0}$ be a sequence in $L^{2}\left((0, T) \times \mathbb{R}^{d}\right)$ which two-scale converges with drift to a limit $U_{0}(t, x, y) \in L^{2}\left((0, T) \times \mathbb{R}^{d} \times \mathbb{T}^{d}\right)$. It satisfies

$$
\lim _{\varepsilon \rightarrow 0}\left\|U_{\varepsilon}\right\|_{L^{2}\left((0, T) \times \mathbb{R}^{d}\right)} \geq\left\|U_{0}\right\|_{L^{2}\left((0, T) \times \mathbb{R}^{d} \times \mathbb{T}^{d}\right)} .
$$

Assume further that

$$
\lim _{\varepsilon \rightarrow 0}\left\|U_{\varepsilon}\right\|_{L^{2}\left((0, T) \times \mathbb{R}^{d}\right)}=\left\|U_{0}\right\|_{L^{2}\left((0, T) \times \mathbb{R}^{d} \times \mathbb{T}^{d}\right)} .
$$

Then, it is said to two-scale converges with drift strongly and it satisfies

$$
\lim _{\varepsilon \rightarrow 0} \int_{0}^{T} \int_{\mathbb{R}^{d}}\left|U_{\varepsilon}(t, x)-U_{0}\left(t, x-\frac{\mathcal{V}}{\varepsilon} t, \frac{x}{\varepsilon}\right)\right|^{2} d x d t=0,
$$

if $U_{0}(t, x, y)$ is smooth, say $U_{0}(t, x, y) \in L^{2}\left((0, T) \times \mathbb{R}^{d} ; C\left(\mathbb{T}^{d}\right)\right)$.

Proof of Theorem 6.1. To begin with, we treat the case of well-prepared initial data, that is, $v^{0}(x)=K u^{0}(x) \in H^{1}\left(\mathbb{R}^{d}\right)$. Later we shall consider more general initial data. The energy equality of (1)-(3) for a time interval $(0, t)$ is

$$
\begin{gathered}
\frac{1}{2}\left[\left\|u_{\varepsilon}(t)\right\|_{L^{2}\left(\Omega_{\varepsilon}\right)}^{2}+\frac{\varepsilon}{K}\left\|v_{\varepsilon}(t)\right\|_{L^{2}\left(\partial \Omega_{\varepsilon}\right)}^{2}\right]+\int_{0}^{t} \int_{\Omega_{\varepsilon}} D_{\varepsilon} \nabla u_{\varepsilon}(s, x) \cdot \nabla u_{\varepsilon}(s, x) d x d s \\
+\frac{\varepsilon}{K} \int_{0}^{t} \int_{\partial \Omega_{\varepsilon}} D_{\varepsilon}^{S} \nabla^{S} v_{\varepsilon}(s, x) \cdot \nabla^{S} v_{\varepsilon}(s, x) d \sigma_{\varepsilon}(x) d s \\
+\frac{\varepsilon \kappa}{\varepsilon^{2}} \int_{0}^{t} \int_{\partial \Omega_{\varepsilon}}\left(u_{\varepsilon}(s, x)-\frac{v_{\varepsilon}}{K}(s, x)\right)^{2} d \sigma_{\varepsilon}(x) d s=\frac{1}{2}\left[\left\|u^{0}\right\|_{L^{2}\left(\Omega_{\varepsilon}\right)}^{2}+\frac{\varepsilon}{K}\left\|v^{0}\right\|_{L^{2}\left(\partial \Omega_{\varepsilon}\right)}^{2}\right]
\end{gathered}
$$

Following the lead of [6] we do not expect a pointwise (in time) strong convergence of the sequence $\left(u_{\varepsilon}, v_{\varepsilon}\right)$. Thus, we integrate once more with respect to time the above energy equality to obtain

$$
\begin{gathered}
\frac{1}{2} \int_{0}^{T}\left[\left\|u_{\varepsilon}(t)\right\|_{L^{2}\left(\Omega_{\varepsilon}\right)}^{2}+\frac{\varepsilon}{K}\left\|v_{\varepsilon}(t)\right\|_{L^{2}\left(\partial \Omega_{\varepsilon}\right)}^{2}\right] d t+\int_{0}^{T} \int_{0}^{t} \int_{\Omega_{\varepsilon}} D_{\varepsilon} \nabla u_{\varepsilon}(s, x) \cdot \nabla u_{\varepsilon}(s, x) d x d s d t \\
+\frac{\varepsilon}{K} \int_{0}^{T} \int_{0}^{t} \int_{\partial \Omega_{\varepsilon}} D_{\varepsilon}^{S} \nabla^{S} v_{\varepsilon}(s, x) \cdot \nabla^{S} v_{\varepsilon}(s, x) d \sigma_{\varepsilon}(x) d s d t \\
+\frac{\varepsilon \kappa}{\varepsilon^{2}} \int_{0}^{T} \int_{0}^{t} \int_{\partial \Omega_{\varepsilon}}\left(u_{\varepsilon}-\frac{v_{\varepsilon}}{K}\right)^{2} d \sigma_{\varepsilon}(x) d s d t=\frac{T}{2}\left(\left\|u^{0}\right\|_{L^{2}\left(\Omega_{\varepsilon}\right)}^{2}+\frac{\varepsilon}{K}\left\|v^{0}\right\|_{L^{2}\left(\partial \Omega_{\varepsilon}\right)}^{2}\right)
\end{gathered}
$$

In the previous section, we established that all terms in the above formula have actually (weak) two-scale limits. Since the corresponding norms are lower semicontinuous with respect to the (weak) two-scale convergence, we deduce

$\liminf _{\varepsilon \rightarrow 0} \frac{1}{2} \int_{0}^{T}\left[\left\|u_{\varepsilon}(t)\right\|_{L^{2}\left(\Omega_{\varepsilon}\right)}^{2}+\frac{\varepsilon}{K}\left\|v_{\varepsilon}(t)\right\|_{L^{2}\left(\partial \Omega_{\varepsilon}\right)}^{2}\right] d t \geq \frac{1}{2}\left(\left|Y^{0}\right|+K\left|\partial \Sigma^{0}\right|\right)\left\|u_{0}\right\|_{L^{2}\left(\mathbb{R}^{d} \times(0, T)\right)}^{2}$, 


$$
\begin{gathered}
\liminf _{\varepsilon \rightarrow 0} \int_{0}^{T} \int_{0}^{t} \int_{\Omega_{\varepsilon}} D_{\varepsilon} \nabla u_{\varepsilon}(s, x) \cdot \nabla u_{\varepsilon}(s, x) d x d s d t \\
\geq \int_{0}^{T} \int_{0}^{t} \int_{\mathbb{R}^{d}} \int_{Y^{0}} D(y)\left|\nabla_{x} u_{0}(s, x)+\nabla_{y}\left(\chi(y) \cdot \nabla_{x} u_{0}(s, x)\right)\right|^{2} d y d x d s d t, \\
\geq \frac{1}{K} \int_{0}^{T} \int_{0}^{t} \int_{\mathbb{R}^{d}} \int_{\partial \Sigma^{0}} D^{S}(y)\left|K G(y) \nabla_{x} u_{0}(s, x)+\nabla_{y}^{S}\left(\omega(y) \cdot \nabla_{x} u_{0}(s, x)\right)\right|^{2} d \sigma(y) d x d s d t, \\
\geq \int_{0}^{T} \int_{0}^{t} \int_{\partial \Omega_{\varepsilon}} D_{\varepsilon}^{S} \nabla^{S} v_{\varepsilon}(s, x) \cdot \nabla^{S} v_{\varepsilon}(s, x) d \sigma_{\varepsilon}(x) d s d t \\
\int_{0}^{T} \int_{0}^{t} \int_{\mathbb{R}^{d}}^{t} \int_{\partial \Sigma^{0}}\left|\left(\chi(y)-K_{\varepsilon \rightarrow 0}^{-1} \omega(y)\right) \cdot \nabla_{x} u_{0}(s, x)\right|^{2} d \sigma(y) d x d s d t .
\end{gathered}
$$

On the other hand we have

$$
\lim _{\varepsilon \rightarrow 0}\left(\left\|u^{0}\right\|_{L^{2}\left(\Omega_{\varepsilon}\right)}^{2}+\frac{\varepsilon}{K}\left\|v^{0}\right\|_{L^{2}\left(\partial \Omega_{\varepsilon}\right)}^{2}\right)=\left|Y^{0}\right|\left\|u^{0}\right\|_{L^{2}\left(\mathbb{R}^{d}\right)}^{2}+\frac{\left|\partial \Sigma^{0}\right|}{K}\left\|v^{0}\right\|_{L^{2}\left(\mathbb{R}^{d}\right)}^{2} .
$$

Summing up those limits and recognizing the formula for $A^{*}$ yields the inequality

$$
\begin{gathered}
\frac{1}{2}\left(\left|Y^{0}\right|+K\left|\partial \Sigma^{0}\right|\right) \\
\left\|u_{0}\right\|_{L^{2}\left(\mathbb{R}^{d} \times(0, T)\right)}^{2}+\int_{0}^{T} \int_{0}^{t} \int_{\mathbb{R}^{d}} A^{*} \nabla_{x} u_{0}(s, x) \cdot \nabla_{x} u_{0}(s, x) d x d s d t \\
\leq \frac{T}{2}\left(\left|Y^{0}\right|\left\|u^{0}\right\|_{L^{2}\left(\mathbb{R}^{d}\right)}^{2}+\frac{\left|\partial \Sigma^{0}\right|}{K}\left\|v^{0}\right\|_{L^{2}\left(\mathbb{R}^{d}\right)}^{2}\right) .
\end{gathered}
$$

However, if we write the same type of time integral of the energy equality for the homogenized equation (14), we get an equality with the same left hand side but a different right hand side

$$
\begin{gathered}
\frac{1}{2}\left[\left|Y^{0}\right|+K\left|\partial \Sigma^{0}\right|\right]\left\|u_{0}\right\|_{L^{2}\left(\mathbb{R}^{d} \times(0, T)\right)}^{2}+\int_{0}^{T} \int_{0}^{t} \int_{\mathbb{R}^{d}} A^{*} \nabla u_{0}(s, x) \cdot \nabla u_{0}(s, x) d x d s d t \\
=\frac{T}{2\left(\left|Y^{0}\right|+K\left|\partial \Sigma^{0}\right|\right)}\left\|\left|Y^{0}\right| u^{0}+\left|\partial \Sigma^{0}\right| v^{0}\right\|_{L^{2}\left(\mathbb{R}^{d}\right)}^{2} .
\end{gathered}
$$

It is easy to check that the two right hand sides in (31) and (32) coincide if and only if

$$
\left\|u^{0}-K^{-1} v^{0}\right\|_{L^{2}\left(\mathbb{R}^{d}\right)}^{2}=0
$$

which is precisely our assumption of well prepared initial data. Therefore, under this assumption we deduce that (31) is not an inequality but rather an equality, which in turn implies that all the previous lower semicontinuity of norm sequences are actually exact convergence. We can thus apply Proposition 6.2 and conclude to a strong convergence (see [6] for details if necessary). 
We now turn to the case of general initial data $\left(u^{0}, v^{0}\right) \in L^{2}\left(\mathbb{R}^{d}\right) \times H^{1}\left(\mathbb{R}^{d}\right)$. Let us consider a small $\delta_{0}>0$, to be chosen precisely at the end of the proof. Due to Lemma 3.1 on a priori estimates, by a contradiction argument, it follows that there exists a time sequence $\left\{\delta_{\varepsilon}\right\}, \delta_{0} / 2 \leq \delta_{\varepsilon} \leq \delta_{0}$, converging to some limit $\bar{\delta}$, $\delta_{0} / 2 \leq \bar{\delta} \leq \delta_{0}$, and a positive constant $C$, which does not depend on $\delta_{0}$ or on $\varepsilon$ (but only on the initial data), such that

$$
\left\{\begin{array}{l}
\left\|u_{\varepsilon}\left(., \delta_{\varepsilon}\right)\right\|_{H^{1}\left(\Omega_{\varepsilon}\right)}^{2} \leq C / \delta_{0}, \quad \varepsilon\left\|v_{\varepsilon}\left(., \delta_{\varepsilon}\right)\right\|_{H^{1}\left(\partial \Omega_{\varepsilon}\right)}^{2} \leq C / \delta_{0}, \\
\varepsilon\left\|K u_{\varepsilon}\left(., \delta_{\varepsilon}\right)-v_{\varepsilon}\left(., \delta_{\varepsilon}\right)\right\|_{L^{2}\left(\partial \Omega_{\varepsilon}\right)}^{2} \leq C \varepsilon^{2} / \delta_{0} .
\end{array}\right.
$$

We now follow an idea of [6] which amounts to consider system (1)-(3) on a smaller time interval $\left(\delta_{\varepsilon}, T\right)$ where $\delta_{\varepsilon} \geq \delta_{0} / 2>0$ is such that the initial data at this time should be almost well-prepared because of parabolic dissipation on the earlier time interval $\left(0, \delta_{\varepsilon}\right)$. We decompose the solution $\left(u_{\varepsilon}, v_{\varepsilon}\right)$ of $(1)-(3)$ as

$$
u_{\varepsilon}(t, x)=u_{1, \varepsilon}(t, x)+u_{2, \varepsilon}(t, x) \quad \text { and } \quad v_{\varepsilon}(t, x)=v_{1, \varepsilon}(t, x)+v_{2, \varepsilon}(t, x),
$$

in such way that the initial data are well prepared for the first problem while the solution of the second one will converge strongly to zero

$$
\begin{cases}\frac{\partial}{\partial t} u_{1, \varepsilon}+\frac{1}{\varepsilon} b_{\varepsilon} \cdot \nabla u_{1, \varepsilon}-\operatorname{div}\left(D_{\varepsilon} \nabla u_{1, \varepsilon}\right)=0 & \text { in }\left(\delta_{\varepsilon}, T\right) \times \Omega_{\varepsilon} \\ -\frac{D_{\varepsilon}}{\varepsilon} \nabla u_{1, \varepsilon} \cdot n=\frac{\partial}{\partial t} v_{1, \varepsilon}+\frac{1}{\varepsilon} b_{\varepsilon}^{S} \cdot \nabla^{S} v_{1, \varepsilon}-\operatorname{div}^{S}\left(D_{\varepsilon}^{S} \nabla^{S} v_{1, \varepsilon}\right) & \text { on }\left(\delta_{\varepsilon}, T\right) \times \partial \Omega_{\varepsilon} \\ -\frac{D_{\varepsilon}}{\varepsilon} \nabla u_{1, \varepsilon} \cdot n=\frac{\kappa}{\varepsilon^{2}}\left[u_{1, \varepsilon}-K^{-1} v_{1, \varepsilon}\right] & \text { on }\left(\delta_{\varepsilon}, T\right) \times \partial \Omega_{\varepsilon} \\ u_{1, \varepsilon}\left(\delta_{\varepsilon}, x\right)=u_{\varepsilon}\left(\delta_{\varepsilon}, x\right), \quad v_{1, \varepsilon}\left(\delta_{\varepsilon}, x\right)=K u_{\varepsilon}\left(\delta_{\varepsilon}, x\right), & \end{cases}
$$

and

$$
\begin{cases}\frac{\partial}{\partial t} u_{2, \varepsilon}+\frac{1}{\varepsilon} b_{\varepsilon} \cdot \nabla u_{2, \varepsilon}-\operatorname{div}\left(D_{\varepsilon} \nabla u_{2, \varepsilon}\right)=0 & \text { in }\left(\delta_{\varepsilon}, T\right) \times \Omega_{\varepsilon}, \\ -\frac{D_{\varepsilon}}{\varepsilon} \nabla u_{2, \varepsilon} \cdot n=\frac{\partial}{\partial t} v_{2, \varepsilon}+\frac{1}{\varepsilon} b_{\varepsilon}^{S} \cdot \nabla^{S} v_{2, \varepsilon}-\operatorname{div}^{S}\left(D_{\varepsilon}^{S} \nabla^{S} v_{2, \varepsilon}\right) & \text { on }\left(\delta_{\varepsilon}, T\right) \times \partial \Omega_{\varepsilon}, \\ -\frac{D_{\varepsilon}}{\varepsilon} \nabla u_{2, \varepsilon} \cdot n=\frac{\kappa}{\varepsilon^{2}}\left[u_{2, \varepsilon}-K^{-1} v_{2, \varepsilon}\right] & \text { on }\left(\delta_{\varepsilon}, T\right) \times \partial \Omega_{\varepsilon}, \\ u_{2, \varepsilon}\left(\delta_{\varepsilon}, x\right)=0, \quad v_{2, \varepsilon}\left(\delta_{\varepsilon}, x\right)=v_{\varepsilon}\left(\delta_{\varepsilon}, x\right)-K u_{\varepsilon}\left(\delta_{\varepsilon}, x\right) . & \end{cases}
$$

Since (35) is similar in structure to (1)-(3), the standard a priori estimate of Lemma 3.1 , together with estimate (33) for the initial data at time $\delta_{\varepsilon}$, yields for any $t \geq \delta_{\varepsilon}$

$$
\left\|u_{2, \varepsilon}(., t)\right\|_{L^{2}\left(\Omega_{\varepsilon}\right)}^{2}+\varepsilon\left\|v_{2, \varepsilon}(., t)\right\|_{L^{2}\left(\partial \Omega_{\varepsilon}\right)}^{2} \leq C \varepsilon^{2} / \delta_{0} .
$$

Thus, the sequence $\left(u_{2, \varepsilon}, v_{2, \varepsilon}\right)$ strongly converges to 0 . The initial data in (34) are well prepared but we cannot apply directly our previous results because the initial time $\delta_{\varepsilon} \neq 0$ is varying with $\varepsilon$. The new difficulty is to prove that the initial data of (34) strongly two scale converge with drift to some limit.

Let us recall the existence of a uniformly bounded extension operator [1] from $H^{1}\left(\Omega_{\varepsilon}\right)$ into $H^{1}\left(\mathbb{R}^{d}\right)$. Thus, the sequence $u_{\varepsilon}\left(\delta_{\varepsilon}, x\right)$ can be thought of being defined in the whole space $\mathbb{R}^{d}$ and, by virtue of (33) it satisfies the bound $\left\|u_{\varepsilon}\left(\delta_{\varepsilon}, x\right)\right\|_{H^{1}\left(\mathbb{R}^{d}\right)}^{2} \leq$ $C / \delta_{0}$. Shifting the sequence does not change its bound, so we have

$$
\left\|u_{\varepsilon}\left(\delta_{\varepsilon}, x+\left(b^{*} / \varepsilon\right) \delta_{\varepsilon}\right)\right\|_{H^{1}\left(\mathbb{R}^{d}\right)}^{2} \leq C / \delta_{0} .
$$


Together with Lemma 6.3 which says that the $L^{2}$-norm of $u_{\varepsilon}\left(\delta_{\varepsilon}, x+\left(b^{*} / \varepsilon\right) \delta_{\varepsilon}\right)$ does not escape at infinity, we deduce from (36) that this sequence is (pre-)compact in $L^{2}\left(\mathbb{R}^{d}\right)$. Therefore, up to a subsequence, $u_{\varepsilon}\left(\delta_{\varepsilon}, x+\left(b^{*} / \varepsilon\right) \delta_{\varepsilon}\right)$ converges strongly to some limit $\tilde{u}^{0}(x)$ in $L^{2}\left(\mathbb{R}^{d}\right)$.

Thus we can use the same arguments as in the case of well-prepared initial data to conclude that $u_{1, \varepsilon}$ and $v_{1, \varepsilon}$ strongly two-scale converge with drift to $\tilde{u}(t, x)$ and $K \tilde{u}(t, x)$ respectively where $\tilde{u}$ satisfies the following limit equation of (34)

$$
\left\{\begin{array}{l}
K_{d} \partial_{t} \tilde{u}=\operatorname{div}_{x}\left(A^{*} \nabla_{x} \tilde{u}\right) \text { in }(\bar{\delta}, T) \times \mathbb{R}^{d} \\
K_{d} \tilde{u}(\bar{\delta}, x)=\left|Y^{0}\right| \tilde{u}^{0}(x)+\left|\partial \Sigma^{0}\right|_{d-1} \tilde{u}^{0}(x), \quad x \in \mathbb{R}^{d}
\end{array}\right.
$$

where $K_{d}=\left|Y^{0}\right|+K\left|\partial \Sigma^{0}\right|_{d-1}$ and $A^{*}$ is given by (15). In particular,

$$
\lim _{\varepsilon \rightarrow 0}\left\|u_{1, \varepsilon}\right\|_{L^{2}\left(\left(\delta_{\varepsilon}, T\right) \times \Omega_{\varepsilon}\right)}^{2}=\left|Y^{0}\right|\|\tilde{u}\|_{L^{2}\left((\bar{\delta}, T) \times \mathbb{R}^{d}\right)}^{2}
$$

and

$$
\lim _{\varepsilon \rightarrow 0} \varepsilon\left\|v_{1, \varepsilon}\right\|_{L^{2}\left(\left(\delta_{\varepsilon}, T\right) \times \partial \Omega_{\varepsilon}\right)}^{2}=K^{2}\left|\partial \Sigma^{0}\right|\|\tilde{u}\|_{L^{2}\left((\bar{\delta}, T) \times \mathbb{R}^{d}\right)}^{2} .
$$

Of course, since $u_{\varepsilon}$ was converging weakly to $u_{0}$, we deduce that $\tilde{u}(t, x)=u_{0}(t, x)$ for $t \geq \bar{\delta}$, and

$$
\begin{aligned}
& \lim _{\varepsilon \rightarrow 0}\left\|u_{\varepsilon}\right\|_{L^{2}\left(\left(\delta_{\varepsilon}, T\right) \times \Omega_{\varepsilon}\right)}^{2}=\left|Y^{0}\right|\left\|u_{0}\right\|_{L^{2}\left((\bar{\delta}, T) \times \mathbb{R}^{d}\right)}^{2} \\
& \lim _{\varepsilon \rightarrow 0} \varepsilon\left\|v_{\varepsilon}\right\|_{L^{2}\left(\left(\delta_{\varepsilon}, T\right) \times \partial \Omega_{\varepsilon}\right)}^{2}=K^{2}\left|\partial \Sigma^{0}\right|\left\|u_{0}\right\|_{L^{2}\left((\bar{\delta}, T) \times \mathbb{R}^{d}\right)}^{2} .
\end{aligned}
$$

Now, let us assume that there is a lack of strong two-scale convergence for $\left(u_{\varepsilon}, v_{\varepsilon}\right)$, namely that the $L^{2}$-norm of this sequence is not continuous (as required by (30) in Proposition 6.2). In other words, either

$$
\lim _{\varepsilon \rightarrow 0}\left\|u_{\varepsilon}\right\|_{L^{2}\left((0, T) \times \Omega_{\varepsilon}\right)}^{2}>\left|Y^{0}\right|\left\|u_{0}\right\|_{L^{2}\left(\mathbb{R}^{d} \times(0, T)\right)}^{2}
$$

or

$$
\lim _{\varepsilon \rightarrow 0} \varepsilon\left\|v_{\varepsilon}\right\|_{L^{2}\left((0, T) \times \partial \Omega_{\varepsilon}\right)}^{2}>K^{2}\left|\partial \Sigma^{0}\right|\left\|u_{0}\right\|_{L^{2}\left(\mathbb{R}^{d} \times(0, T)\right)}^{2} .
$$

Lemma 3.1 implies that $\left\|u_{\varepsilon}(t)\right\|_{L^{2}\left(\Omega_{\varepsilon}\right)}^{2}+\varepsilon\left\|v_{\varepsilon}(t)\right\|_{L^{2}\left(\partial \Omega_{\varepsilon}\right)}^{2} \leq C$ uniformly in time. So, we can find a small $\delta_{0}>0$ such that, the same strict inequalities hold true on a smaller time interval, namely, either

$$
\liminf _{\varepsilon \rightarrow 0}\left\|u_{\varepsilon}\right\|_{L^{2}\left((\delta, T) \times \Omega_{\varepsilon}\right)}^{2}>\left|Y^{0}\right|\left\|u_{0}\right\|_{L^{2}\left((0, T) \times \mathbb{R}^{d}\right)}^{2} \quad \text { for any } 0<\delta<\delta_{0},
$$

or

$$
\liminf _{\varepsilon \rightarrow 0} \varepsilon\left\|v_{\varepsilon}\right\|_{L^{2}\left((\delta, T) \times \partial \Omega_{\varepsilon}\right)}^{2}>K^{2}\left|\partial \Sigma^{0}\right|\left\|u_{0}\right\|_{L^{2}\left((0, T) \times \mathbb{R}^{d}\right)}^{2} \quad \text { for any } 0<\delta<\delta_{0} .
$$

Obviously, (41) or (42) contradicts (38). Therefore, there must be continuity of the $L^{2}$-norm of $\left(u_{\varepsilon}, v_{\varepsilon}\right)$ and both $(39)$ and (40) must be equalities. Thus, we have proved the strong two-scale convergence with drift in case of a general initial data.

Lemma 6.3 Let $u_{\varepsilon}(t, x)$ be the solution of (1)-(3). For any $\delta>0$ there is $R(\delta)>0$ such that, for any $t \in[0, T]$,

$$
\left\|u_{\varepsilon}\left(x+\frac{b^{*}}{\varepsilon} t, t\right)\right\|_{L^{2}\left(\Omega_{\varepsilon} \cap\{x \geq R(\delta)\}\right)} \leq \delta .
$$


Proof. We again follow an idea of [6]. Let $\phi \in C^{\infty}(\mathbb{R})$ be a cut-off function such that $0 \leq \phi(r) \leq 1, \phi=0$ for $r \leq 1, \phi=1$ for $r \geq 2$. For $x \in \mathbb{R}^{d}$, denote $\phi_{R}(x)=\phi(|x| / R)$ and $\phi_{R}^{\varepsilon}(t, x)=\phi_{R}\left(x-b^{*} t / \varepsilon\right)$. In the variational formulation (4) of system (1)-(2) we take the test function $\left\{u_{\varepsilon}(t, x) \phi_{R}^{\varepsilon}(t, x), v_{\varepsilon}(t, x) \phi_{R}^{\varepsilon}(t, x)\right\}$ and we integrate by parts the time and convective derivatives (calculations are shown one term at a time)

$$
\begin{gathered}
2 \int_{0} \int_{\Omega_{\varepsilon}} \frac{\partial u_{\varepsilon}}{\partial t}(s, x) u_{\varepsilon}(s, x) \phi_{R}^{\varepsilon}(s, x) d s d x=\int_{0}^{t} \int_{\Omega_{\varepsilon}} \frac{b^{*}}{\varepsilon} \cdot \nabla \phi_{R}^{\varepsilon}(s, x)\left(u_{\varepsilon}(s, x)\right)^{2} d s d x \\
+\int_{\Omega_{\varepsilon}} \phi_{R}^{\varepsilon}(t, x)\left(u_{\varepsilon}(t, x)\right)^{2} d x-\int_{\Omega_{\varepsilon}} \phi_{R}(x)\left(u^{0}(x)\right)^{2} d x, \\
2 \int_{0 \Omega_{\varepsilon}}^{t} \frac{b_{\varepsilon}}{\varepsilon} \cdot \nabla u_{\varepsilon}(s, x) u_{\varepsilon}(s, x) \phi_{R}^{\varepsilon}(s, x) d s d x=-\int_{0}^{t} \int_{\Omega_{\varepsilon}} \frac{b_{\varepsilon}}{\varepsilon} \cdot \nabla \phi_{R}^{\varepsilon}(s, x)\left(u_{\varepsilon}(s, x)\right)^{2} d s d x, \\
\frac{2 \varepsilon}{K} \int_{0}^{t} \int_{\partial \Omega_{\varepsilon}}^{t} \frac{\partial v_{\varepsilon}}{\partial t} \phi_{R}^{\varepsilon} v_{\varepsilon} d s d \sigma(x)=\frac{b^{*}}{K} \cdot \nabla \phi_{R}^{\varepsilon}\left(v_{\varepsilon}\right)^{2} d s d \sigma(x) \\
+\frac{\varepsilon}{K} \int_{\partial \Omega_{\varepsilon}} \phi_{R}^{\varepsilon}\left(v_{\varepsilon}\right)^{2} d \sigma(x)-\frac{\varepsilon}{K} \int_{\partial \Omega_{\varepsilon}} \phi_{R}(x)\left(v^{0}(x)\right)^{2} d \sigma(x), \\
\int_{0}^{t} \int_{\partial \Omega_{\varepsilon}} b_{\varepsilon}^{S} \cdot \nabla^{S} v_{\varepsilon} v_{\varepsilon} \phi_{R}^{\varepsilon} d s d \sigma(x)=-\frac{1}{K} \int_{0}^{t} \int_{\partial \Omega_{\varepsilon}}\left(v_{\varepsilon}\right)^{2} b_{\varepsilon}^{S} \cdot \nabla^{S} \phi_{R}^{\varepsilon} d s d \sigma(x) .
\end{gathered}
$$

This yields

$$
\begin{aligned}
& \quad \frac{1}{2} \int_{\Omega_{\varepsilon}} \phi_{R}^{\varepsilon}(t, x)\left(u_{\varepsilon}(t, x)\right)^{2} d x+\int_{0} \int_{\Omega_{\varepsilon}} D_{\varepsilon} \nabla u_{\varepsilon} \cdot \nabla u_{\varepsilon} \phi_{R}^{\varepsilon} d s d x \\
& +\frac{\varepsilon}{2 K} \int_{\partial \Omega_{\varepsilon}} \phi_{R}^{\varepsilon}(t, x)\left(v_{\varepsilon}(t, x)\right)^{2} d \sigma(x)+\frac{\varepsilon}{K} \int_{0}^{t} \int_{\partial \Omega_{\varepsilon}} D_{\varepsilon}^{S} \nabla^{S} v_{\varepsilon} \cdot \nabla^{S} v_{\varepsilon} \phi_{R}^{\varepsilon} d s d \sigma(x) \\
& +\frac{\kappa \varepsilon}{\varepsilon^{2}} \int_{0}^{t} \int_{\partial \Omega_{\varepsilon}} \phi_{R}^{\varepsilon}\left(u_{\varepsilon}-K^{-1} v_{\varepsilon}\right)^{2} d s d \sigma(x) \\
& =-\int_{0}^{t} \int_{\Omega_{\varepsilon}} u_{\varepsilon} D_{\varepsilon} \nabla u_{\varepsilon} \cdot \nabla \phi_{R}^{\varepsilon} d s d x-\frac{\varepsilon}{K} \int_{0}^{t} \int_{\partial \Omega_{\varepsilon}} v_{\varepsilon} D_{\varepsilon}^{S} \nabla^{S} v_{\varepsilon} \cdot \nabla^{S} \phi_{R}^{\varepsilon} d s d \sigma(x) \\
& +\int_{0}^{t} \frac{1}{\Omega_{\varepsilon}}\left(b_{\varepsilon}-b^{*}\right) \cdot \nabla \phi_{R}^{\varepsilon}\left(u_{\varepsilon}\right)^{2} d s d x+\frac{1}{2} \int_{\Omega_{\varepsilon}} \phi_{R}(x)\left(u^{0}(x)\right)^{2} d x \\
& +\int_{0}^{t} \int_{\partial \Omega_{\varepsilon}} \frac{1}{K}\left(b_{\varepsilon}^{S}-b^{*}\right) \cdot \nabla^{S} \phi_{R}^{\varepsilon}\left(v_{\varepsilon}\right)^{2} d s d \sigma(x)+\frac{\varepsilon}{2 K} \int_{\partial \Omega_{\varepsilon}} \phi_{R}(x)\left(v^{0}(x)\right)^{2} d \sigma(x)
\end{aligned}
$$

Due to the a priori estimate of Lemma 3.1 and the definition of $\phi_{R}$, the first and second integrals on the right hand side of (43) are uniformly bounded by $C / R$. The 
terms involving the initial data $\left(u^{0}(x), v^{0}(x)\right)$ do not depend on $\varepsilon$ and tend to zero as $R$ tends to $\infty$. To get a bound on the remaining (convective) terms on the right hand side of (43), we rely again on the auxiliary problem (27) which allows us to remove the $\varepsilon^{-1}$ singularity of the convective terms

$$
\begin{aligned}
& \int_{0}^{t} \int_{\Omega_{\varepsilon}} \frac{1}{\varepsilon}\left(b_{\varepsilon}-b^{*}\right) \cdot \nabla \phi_{R}^{\varepsilon}\left(u_{\varepsilon}\right)^{2} d s d x+\int_{0}^{t} \int_{\partial \Omega_{\varepsilon}} \frac{1}{K}\left(b_{\varepsilon}^{S}-b^{*}\right) \cdot \nabla^{S} \phi_{R}^{\varepsilon}\left(v_{\varepsilon}\right)^{2} d s d \sigma(x) \\
= & \varepsilon \sum_{i=1}^{d} \int_{0}^{t} \int_{\Omega_{\varepsilon}} \nabla \alpha_{i}^{\varepsilon} \cdot \nabla\left(\left(u_{\varepsilon}\right)^{2} \partial_{x_{i}} \phi_{R}^{\varepsilon}\right) d s d x+K \int_{0}^{t} \int_{\partial \Omega_{\varepsilon}} \nabla \phi_{R}^{\varepsilon} \cdot\left(b^{*}-b_{\varepsilon}^{S}\right)\left(\left(u_{\varepsilon}\right)^{2}-K^{-2}\left(v_{\varepsilon}\right)^{2}\right) d s d \sigma(x) .
\end{aligned}
$$

Since $\left(u_{\varepsilon}^{2}-K^{-2} v_{\varepsilon}^{2}\right)=\left(u_{\varepsilon}+K^{-1} v_{\varepsilon}\right)\left(u_{\varepsilon}-K^{-1} v_{\varepsilon}\right)$ and $\varepsilon \nabla \alpha_{i}^{\varepsilon}(x)=\left(\nabla_{y} \alpha_{i}\right)(x / \varepsilon)$, the a priori estimates of Lemma 3.1 imply that (44) is uniformly bounded by $C / R$ too, which yields the desired statement.

\section{Numerical study}

This section is devoted to the numerical computation of the cell problems and of the homogenized coefficients, given by Proposition 4.3, and to the study of their variations according to various parameters in the microscopic model. Our numerical simulations are performed with the FreeFem ++ package [27]. We have used Lagrange P1 finite elements with 33586 vertices (degrees of freedom) with characteristic Galerkin method for the convective term. The periodicity cell is the unit square and the solid obstacle is a disk. Table 1 gives the adimensionalized values of the parameters utilized in our simulations.

\begin{tabular}{|l|l|}
\hline Parameters & Values \\
\hline Radius of the obstacle $r$ & 0.2 \\
\hline Equilibrium constant $K$ & 1 \\
\hline Porosity : $\left|Y^{0}\right|=1-r^{2} \pi$ & 0.874357 \\
\hline Tortuosity : $\left|\partial \Sigma^{0}\right|=2 \pi r$ & 1.25664 \\
\hline$K_{d}$ factor : $\left|Y^{0}\right|+K\left|\partial \Sigma^{0}\right|$ & 2.13099 \\
\hline Surface velocity $b^{S}$ & 0 \\
\hline Mean velocity $\int_{Y^{0}} b(y) d y$ & $\left(0.0385,-2.67 \times 10^{-5}\right)$ \\
\hline Adsorption rate $\kappa_{0}$ & 1 \\
\hline Bulk molecular diffusion $D$ & 1 \\
\hline Surface molecular diffusion $D^{S}$ & 1 \\
\hline
\end{tabular}

Table 1: Parameter values

The velocity profile $b(y)$ is generated by solving the following Stokes problem in the fluid part $Y^{0}$ of the unit cell.

$$
\begin{cases}\nabla_{y} p-\Delta_{y} b=e_{1} & \text { in } Y^{0} \\ \operatorname{div}_{y} b=0 & \text { in } Y^{0} \\ b=0 & \text { on } \partial \Sigma^{0} \\ p, b & Y-\text { periodic. }\end{cases}
$$

The drift velocity $b^{*}$ is then calculated using (13): we found $b^{*}=(0.0180,-1.25 *$ $\left.10^{-5}\right)$. 


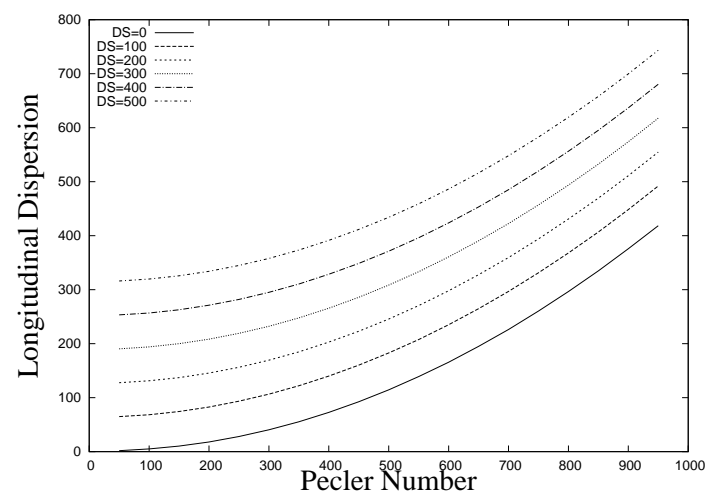

Figure 1: Behavior of the longitudinal dispersion with respect to $P e_{l o c}$ for various values of the surface molecular diffusion $D^{S}$.

In a first experiment we study the behavior of the longitudinal dispersion with respect to the local Péclet number $P e_{l o c}$ (we simply multiply the velocity field $b(y)$ by an increasing factor) for various values of $D^{S}$ (see Figure 1). As can be expected the dispersion increases with $P e_{l o c}$. Clearly the dispersion increases with $D^{S}$ too. However, as shown by Figure 2, the dispersion reaches a limit as $D^{S}$ goes to infinity. This can be explained formally by the fact that, in such a case, the cell solution satisfies in the limit that $\left(\omega_{i}+K y_{i}\right)$ is constant on the pore surface $\partial \Sigma^{0}$. In this limit, the bulk correctors $\chi_{i}$ satisfy the following limit problem

$$
\begin{cases}b(y) \cdot \nabla_{y} \chi_{i}-\operatorname{div}_{y}\left(D\left(\nabla_{y} \chi_{i}+e_{i}\right)\right)=\left(b^{*}-b\right) \cdot e_{i} & \text { in } Y^{0} \\ -D\left(\nabla_{y} \chi_{i}+e_{i}\right) \cdot n+K b_{i}^{*}= & \\ \kappa\left(\chi_{i}+y_{i}-\left|\partial \Sigma^{0}\right|^{-1} \int_{\partial \Sigma^{0}}\left(\chi_{i}+y_{i}\right) d \sigma(y)\right) & \text { on } \partial \Sigma^{0} \\ y \rightarrow \chi_{i}(y) & Y-\text { periodic. }\end{cases}
$$
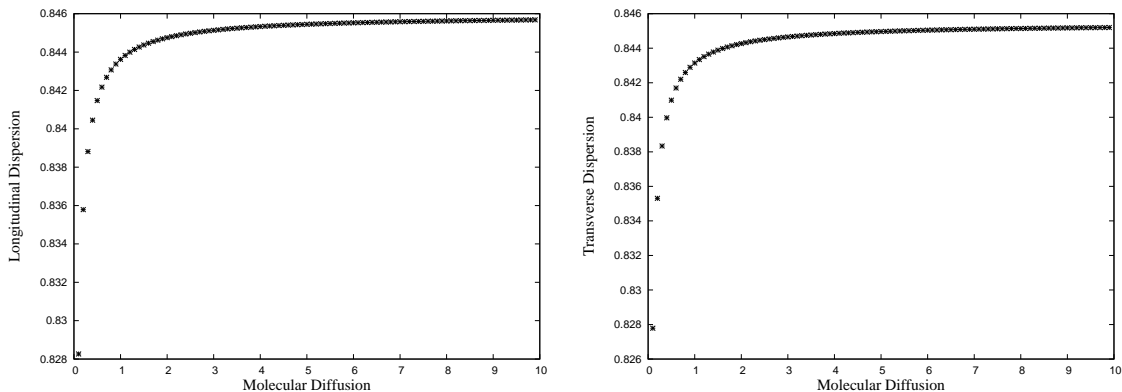

Figure 2: Behavior of the effective dispersion with respect to $D^{S}$ : longitudinal dispersion (left), transverse dispersion (right).

In a second experiment we study the behavior of the longitudinal dispersion with respect to the reaction rate $\kappa$. Interestingly enough, we observe an inversion phenomenon on the bulk corrector $\chi_{1}$ (see Figure 3 where the grey scale goes from smaller values in white to larger values in black). A similar inversion is exhibited by $\chi_{2}$ too. However, this inversion phenomenon doesn't appear in the absence of surface molecular diffusion, i.e., when $D^{S}=0$. For a given positive value of $D^{S}$, increasing $\kappa$ implies that it may be more favorable for the solute to "travel" close to the pore surface by using the surface diffusion. Therefore, the solid pores may 
be seen as obstacles for small $\kappa$ or attractors for large $\kappa$. It is probably easier to interpret this inversion phenomenon on the "reconstructed" solution $\left(\chi_{1}(y)+y_{1}\right)$ rather than on just on the cell solution $\chi_{1}(y)$ (see Figure 4$)$. Indeed, $\left(\chi_{1}(y)+y_{1}\right)$ corresponds to a local linearization of the two first terms of the ansatz of $u_{\varepsilon}$ as given in Proposition 4.3. As the reaction rate increases, the isolines of this reconstructed concentration start to grace off the obstacle, which implies that the orthogonal current lines of the concentration flux are more and more attracted by the obstacle. It should be remarked here that this is a handicap of performing only $2-d$ numerical simulations. Indeed, it is only in $3-d$ that both the fluid phase and the solid structure can be connected, which only permits a fair comparison between surface and bulk molecular diffusions.
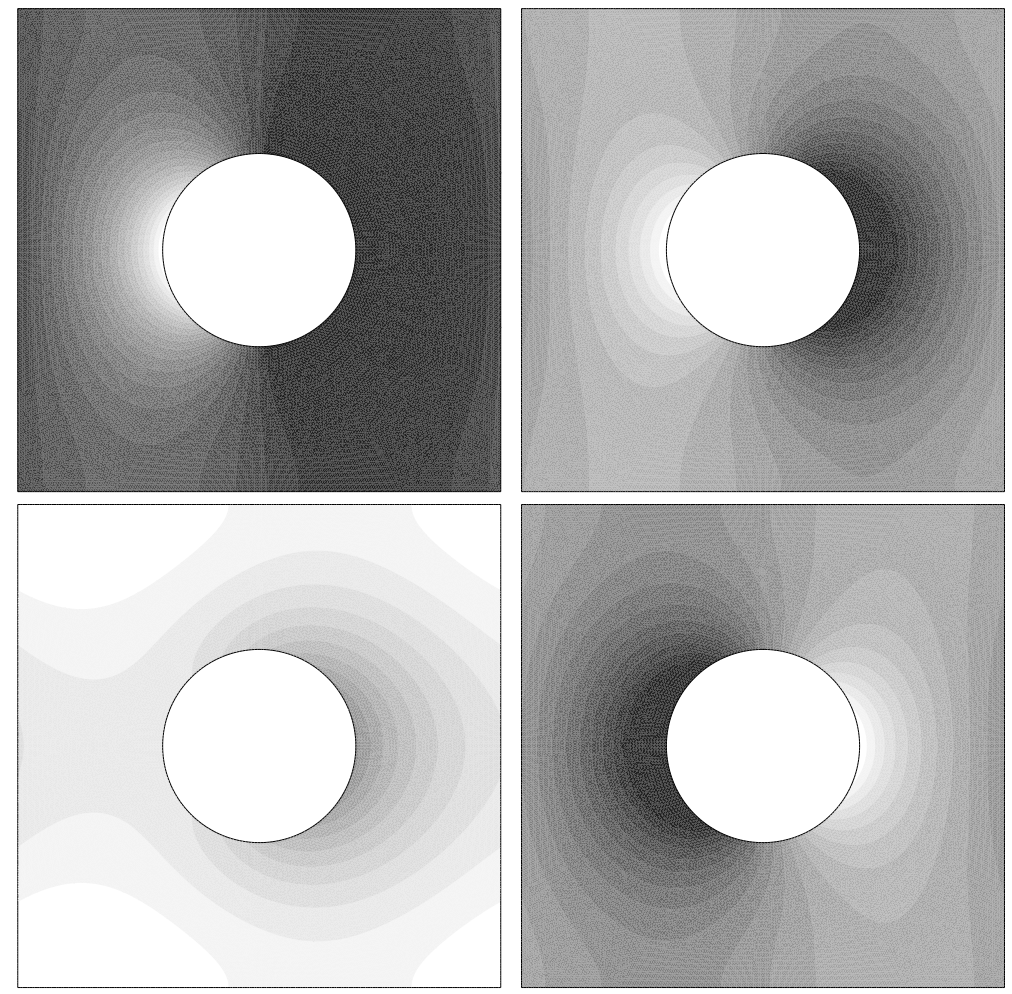

Figure 3: The cell solution $\chi_{1}$ : Left top, reference value $\kappa=\kappa^{0}$; Right top $\kappa=5 \kappa^{0}$; Left bottom $\kappa=6 \kappa^{0}$; Right bottom $\kappa=8 \kappa^{0}$.

In Figures 5 and 6 we plot the dispersion in two asymptotic regimes: $\kappa \rightarrow 0$ and $\kappa \rightarrow \infty$. Once again when $\kappa \rightarrow \infty$ we get an asymptote for the dispersion, corresponding to a limit cell problem where $K \chi_{i}=\omega_{i}$. In this limit, the corresponding system satisfied by the bulk corrector $\chi_{i}$ is

$$
\left\{\begin{array}{cl}
b(y) \cdot \nabla_{y} \chi_{i}-\operatorname{div}_{y}\left(D\left(\nabla_{y} \chi_{i}+e_{i}\right)\right)=\left(b^{*}-b\right) \cdot e_{i} & \text { in } Y^{0} \\
-K^{-1} D\left(\nabla_{y} \chi_{i}+e_{i}\right) \cdot n+\left(b^{*}-b^{S}\right) \cdot e_{i}= & \\
b^{S}(y) \cdot \nabla_{y}^{S} \chi_{i}-\operatorname{div}_{y}^{S}\left(D^{S}\left(\nabla_{y}^{S} \chi_{i}+e_{i}\right)\right) & \text { on } \partial \Sigma^{0} \\
y \rightarrow \chi_{i}(y) & Y-\text { periodic. }
\end{array}\right.
$$

Of course, the transverse dispersion is always smaller than the longitudinal dispersion. Figures 5 and 6 show the blow-up behavior of both longitudinal and transverse dispersions when $\kappa \rightarrow 0$. This is due to the ill-posedness of the cell problem (17) in the limit $\kappa \rightarrow 0$. 

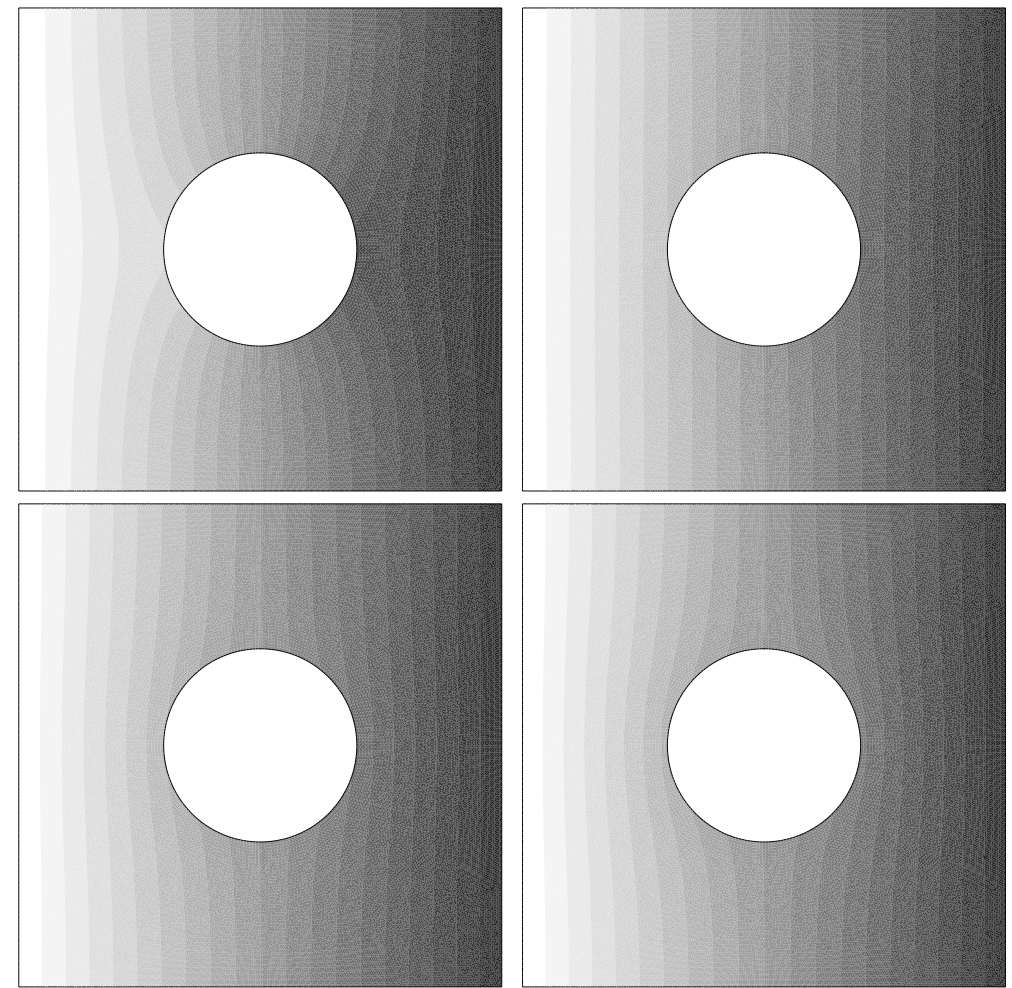

Figure 4: The reconstructed solution $\chi_{1}(y)+y_{1}$ : Left top, Reference value $\kappa=\kappa^{0}$; Right top $\kappa=6 \kappa^{0} ;$ Left bottom $\kappa=12 \kappa^{0}$; Right bottom $\kappa=19 \kappa^{0}$
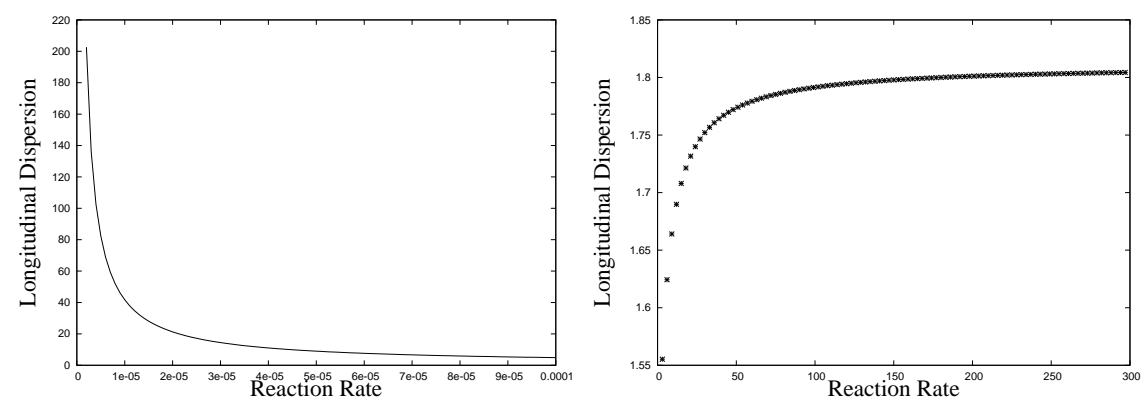

Figure 5: Behavior of the longitudinal dispersion with respect to $\kappa$ : when $\kappa$ goes to 0 (left), when $\kappa$ goes to infinity (right).

Acknowledgements. This work was partially supported by the GNR MOMAS (Modélisation Mathématique et Simulations numériques liées aux problèmes de gestion des déchets nucléaires) (PACEN/CNRS, ANDRA, BRGM, CEA, EDF, IRSN) and by the Chair "Mathematical modelling and numerical simulation, F-EADS Ecole Polytechnique - INRIA". G. A. is a member of the DEFI project at INRIA Saclay Ile-de-France. 

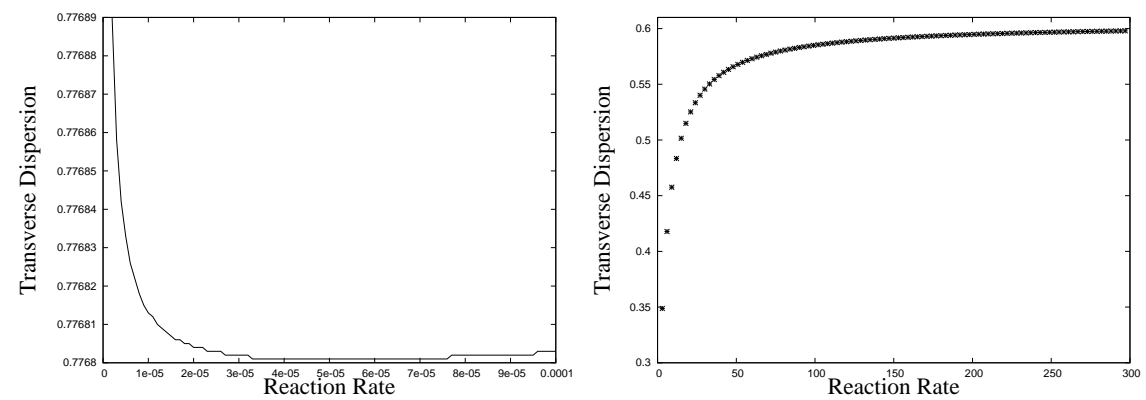

Figure 6: Behavior of the transverse dispersion with respect to $\kappa$ : when $\kappa$ goes to 0 (left), when $\kappa$ goes to infinity (right).

\section{References}

[1] Acerbi E., Chiado Piat V., Dal Maso G., Percivale D. An extension theorem from connected sets, and homogenization in general periodic domains, Nonlinear Anal., Vol 18 (1992), pp.481-496.

[2] Allaire G. Homogenization and two-scale convergence, SIAM J. Math. Anal., Vol 23 No.6 (1992), pp.1482-1518.

[3] Allaire G. Periodic homogenization and effective mass theorems for the Schrödinger equation. Quantum Transport-Modelling, analysis and asymptotics, Ben Abdallah N. and Frosali G. eds., Lecture Notes in Mathematics 1946, Springer (2008), pp.1-44.

[4] Allaire G., Brizzi R., Mikelić A., Piatnitski A. Two-scale expansion with drift approach to the Taylor dispersion for reactive transport through porous media, Chemical Engineering Science, Vol 65 (2010), pp.2292-2300

[5] Allaire G., Damlamian A., Hornung U. Two-scale convergence on periodic surfaces and applications, Proceedings of the International Conference on Mathematical Modelling of Flow through Porous Media (May 1995), Bourgeat A. et al. eds., World Scientific Pub., Singapore (1996), pp.15-25.

[6] Allaire G., Mikelić A., Piatnitski A. Homogenization approach to the dispersion theory for reactive transport through porous media, SIAM J. Math. Anal., Vol 42 No.1 (2010), pp.125-144.

[7] G. Allaire, R. Orive, Homogenization of periodic non self-adjoint problems with large drift and potential, COCV 13, pp.735-749 (2007).

[8] Allaire G., Raphael A.L. Homogenization of a convection diffusion model with reaction in a porous medium, C. R. Math. Acad. Sci. Paris, Vol 344 No.8 (2007), pp.523-528.

[9] Auriault J.L., Adler P.M. Taylor dispersion in porous media : Analysis by multiple scale expansions, Advances in Water Resources, Vol 18 (1995), pp.217-226.

[10] Bear J., Bachmat Y. Introduction to Modeling of Transport Phenomena in Porous Media. Theory and Applications of Transport in Porous Media, Kluwer Academic Publishers, The Netherlands (1991).

[11] Brézis H. Analyse Fonctionelle, Théorie et applications, Collection Mathématiques Appliquées pour la Maîtrise, Masson, Paris (1983). 
[12] Canon E., Bensmina H., Valentin P. On the modelling of generalized Taylor Aris Dispersion in Chromatographs via Multiple Scales Expansions, Transport in Porous Media, Vol 36 (1999), pp.307-339.

[13] Donato P., Piatnitski A. Averaging of nonstationary parabolic operators with large lower order terms, Multi Scale Problems and Asymptotic Analysis, GAKUTO International Series, Math. Sci. Appl., Vol 24 (2005), pp.153-165.

[14] van Duijn C.J., Pop I.S. Crystal dissolution and precipitation in porous media: pore scale analysis, J. Reine Angew. Math., Vol 577 (2004), pp.171-211.

[15] Evans L.C. Partial Differential Equations, Graduate Studies in Mathematics, Vol 19, American Mathematical Society, Providence, RI (1998).

[16] Greenkorn R.A. Flow phenomena in porous media : fundamentals and applications in petroleum, water, and food product, Marcel Dekker, New York (1984).

[17] Hornung U. Homogenization and porous media, Interdisciplinary Applied Mathematics Series, Vol 6, Springer Verlag (1997).

[18] Hornung U., Jäger W. Diffusion, convection, adsorption, and reaction of chemicals in porous media, J. Diff. Eq., Vol 92 (1991), pp.199-225.

[19] Hutridurga H. PhD thesis, in preparation, Ecole Polytechnique.

[20] Jikov V.V., Kozlov S.M., Oleinik O.A. Homogenization of differential operators and integral functionals, Springer, Berlin (1994).

[21] Marusic-Paloka E., Piatnitski A. Homogenization of a nonlinear convectiondiffusion equation with rapidly oscillating co-efficients and strong convection, Journal of London Math. Soc., Vol 72 No.2 (2005), pp.391-409.

[22] Mauri R. Dispersion, convection and reaction in porous media, Phys. Fluids A, Vol 3 (1991), pp.743-755.

[23] Mei C C. Method of homogenization applied to dispersion in porous media, Transport in Porous Media, Vol 9 (1992), pp.261-274.

[24] Nguetseng G. A general convergence result for a functional related to the theory of homogenization, SIAM J. Math. Anal., Vol 20 No.3 (1989), pp.608-623.

[25] Neuss-Radu M. Some extensions of two-scale convergence, C. R. Acad. Sci. Paris Sr. I Math., Vol 322 No.9 (1996), pp.899-904.

[26] Papanicolaou G.C. Diffusion in random media, Surveys in applied mathematics, Vol 1, Plenum, New York (1995), pp.201-253.

[27] Pironneau O., Hecht F., Le Hyaric A. FreeFem++ version 2.15-1, http://www.freefem.org/ff++/

[28] Rubinstein J., Mauri R. Dispersion and convection in porous media, SIAM J. Appl. Math., Vol 46 (1986), pp.1018-1023.

[29] Taylor G.I. Dispersion of soluble matter in solvent flowing slowly through a tube, Proc. Royal Soc. A, Vol 219 (1953), pp.186-203. 